\title{
Paragrassmann Extensions of the Virasoro Algebra
}

\author{
A.T.Filippov, A.P. Isaev ${ }^{\dagger}$ and A.B.Kurdikov \\ Laboratory of Theoretical Physics, JINR, \\ Head Post Office, P.O. Box 79 \\ Dubna, SU-101 000 Moscow, Russia
}

\begin{abstract}
The paragrassmann differential calculus is briefly reviewed. Algebras of the transformations of the para-superplane preserving the form of the parasuperderivative are constructed and their geometric meaning is discussed. A new feature of these algebras is that they contain generators of the automorphisms of the paragrassmann algebra (in addition to Ramond-Neveu-Schwarz - like conformal generators). As a first step in analyzing these algebras we introduce more tractable multilinear algebras not including the new generators. In these algebras there exists a set of multilinear identities based on the cyclic polycommutators. Different possibilities of the closure are therefore admissible. The central extensions of the algebras are given. Their number varies from 1 to $\left[\frac{p+1}{2}\right]$ depending on the form of the closure chosen. Finally, simple explicit examples of the paraconformal transformations are given.
\end{abstract}

*Address until Jan.14, 1993: Yukawa Institute of Theor. Phys., Kyoto Univ., Kyoto 606, Japan e-mail: filippov@jpnyitp@ @jpnyitp.yukawa.kyoto-u.ac.jp filippov@theor.jinrc.dubna.su

$\dagger$ e-mail address: isaevap@theor.jinrc.dubna.su

$\ddagger$ e-mail address: kurdikov@theor.jinrc.dubna.su 


\section{Introduction}

Different extensions of the Virasoro algebra are useful in formulating two-dimensional quantum conformal field theories with certain additional symmetries [1]. Such extensions are generated by the stress-energy tensor $T$ and some currents corresponding to the additional symmetries. For example, if we add the Kac-Moody currents $J$ of the conformal weight 1 , the result will be the well-known semi-direct sum of the Virasoro and Kac-Moody algebras with Sugawara-type relations between $J$ and $T$. Adding instead a fermionic current having the weight $3 / 2$ we get the Ramond-NeveuSchwarz algebra (RNS). $S O(N)$ or $S U(N)$-invariant extensions and $N=2,3,4$ super-extensions of the RNS algebra have been considered in the context of $2 \mathrm{D}$ conformal field theories in [2].

Introducing currents $W_{N}$ with integer weights $N \geq 3$ gives rise to the $W_{N^{-}}$ algebras of A. Zamolodchikov which have demonstrated their usefulness in last years. So it seems that a most interesting new possibility is to look for further extensions by trying to add currents with fractional conformal weights and, in particular, parafermionic currents of weights $(p+2) /(p+1)(p$ is a positive integer which is, in fact, the order of parastatistics or the degree of nilpotency of an underlying paragrassmann algebra). A construction of such an extension of the Virasoro algebra is the subject of the present paper.

Attempts to realize this possibility had already been made. First of all, we have to mention the work of V. Fateev and A. Zamolodchikov [3] where a system with

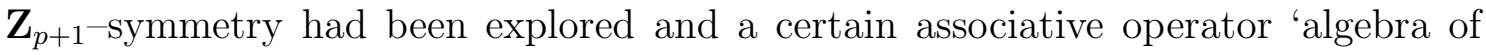
parafermionic currents' has been constructed. That algebra, although, may hardly be regarded as a paragrassmann extension of the Virasoro-RNS algebra since it does not reproduce the RNS algebra for $p=1$.

Another attempt, stimulated by a para-supersymmetric quantum mechanics of V. Rubakov and V. Spiridonov [4], had been undertaken by S. Durand et.al. [5]. The formulation of Ref. [5] was based on a paragrassmann calculus defined in the frame of the Green representation for the paragrassmann algebra also known as the Green ansatz [6]. In a later paper [7] S. Durand is using the paragrassmann calculus developed 4 in Ref. [8] to derive interesting identities involving VirasoroRNS - like generators. Some of these identities look like plausible ingredients of a para-extension of the superconformal algebra but their relation to any symmetry transformations remains unclear. A relation of similar identities to the conformal algebra in two dimensions, also hinting at possible generalizations of the superconformal symmetry, has been found by T.Nakanishi [10].

All this motivates our present attempt to find a systematic para-generalization of the superconformal and Virasoro-RNS algebras. To clarify the logic of our paper we first recall the classical cases $p=0$ and $p=1$ in a framework we are going to generalize.

\section{$p=0$ ( Virasoro case )}

The space is simply the complex plane with the coordinate $z$. Let $F=F(z)$ be

\footnotetext{
${ }^{1}$ As we have learned after submitting our paper [8] to the HEP-TH database, some ingredients of this calculus for one variable were earlier presented in Ref. [9].
} 
an analytic function of $z$, and consider an analytic map

$$
z \mapsto \tilde{z}(z), \quad F(z) \mapsto \tilde{F} \equiv F(\tilde{z}(z)) .
$$

The trivial identity

$$
\frac{\partial}{\partial z} \tilde{F}=\tilde{z}^{\prime} \frac{\partial}{\partial \tilde{z}} \tilde{F}
$$

(prime will always denote the derivative with respect toz) means that the old and new derivatives are proportional for any function $\tilde{z}$, or in other words, any transformation (1.1) will be conformal, i.e. 'preserving the form of the derivative'. The group of the conformal transformations will be denoted by CON.

Passing to the infinitesimal form of the transformation (1.1), $\tilde{z}=z+\lambda \omega(z) \quad(\lambda$ is a small number), and defining its generator $T(\omega)$ by

$$
\tilde{F}=(1+\lambda T(\omega)) F,
$$

one easily sees that $T(\omega)=\omega \partial_{z}$. The Lie algebra generated by $T(\omega)$ is defined by the commutators

$$
[T(\omega), T(\psi)]=T\left(\omega \psi^{\prime}-\omega^{\prime} \psi\right)
$$

and is called the conformal algebra, Con in our notation. It coincides with the whole algebra of vector fields on the complex plane and its (unique) central extension is the standard Virasoro algebra denoted by Vir.

There is a simple generalization of the construction when $F$ are not ordinary functions but conformal fields of weight $\Delta$. In this case the transformation rule is

$$
F_{\Delta}(z) \mapsto \tilde{F}_{\Delta} \equiv\left(\tilde{z}^{\prime}\right)^{\Delta} F_{\Delta}(\tilde{z})
$$

and the generators have the form $T(\omega)=\omega \partial_{z}+\Delta \omega^{\prime}$. Their Lie algebra coincides with (1.4).

$p=1$ ( RNS-case).

Now the space is a complex superplane with coordinates $z$ and $\theta, \theta^{2}=0$. In fact, for a precise formulation we need a Grassmann algebra of more than one variable. One of them will be specified as $\theta$ while the rest will be referred as 'other thetas'. A super-analytic map is

$$
\begin{aligned}
z \mapsto \tilde{z} & =Z_{0}+\theta Z_{1}, \\
\theta \mapsto \tilde{\theta} & =\Theta_{0}+\theta \Theta_{1}, \quad \tilde{\theta}^{2}=0,
\end{aligned}
$$

where $Z_{i}$ and $\Theta_{i}$ are functions of $z$ and of 'other thetas' with needed Grassmann parities. This map transforms functions $F=F(z, \theta)$ into $\tilde{F} \equiv F(\tilde{z}, \tilde{\theta})$.

The fractional derivative of order $1 / 2$ can be defined as

$$
\mathcal{D}=\partial_{\theta}+\theta \partial_{z}, \quad \mathcal{D}^{2}=\partial_{z},
$$

due to the Grassmann relations $\theta^{2}=0=\partial_{\theta}^{2},\left\{\partial_{\theta}, \theta\right\}_{+}=1$. The super-analytic maps (1.6), (1.7) are called superconformal transformations when the superderivative transforms homogeneously (see e.g. [11], [12]). This requirement, similar to (1.2), can be written in the form

$$
\mathcal{D} \tilde{F}=\Phi \tilde{\mathcal{D}} \tilde{F}
$$


and leads to certain restrictions on the parameter functions, namely,

$$
\Phi=\mathcal{D} \tilde{\theta}, \mathcal{D} \tilde{z}=\mathcal{D}(\tilde{\theta}) \tilde{\theta}
$$

or, in the component notation,

$$
\begin{aligned}
& Z_{1}=\Theta_{1} \Theta_{0} \\
& Z_{0}^{\prime}=\Theta_{0}^{\prime} \Theta_{0}+\left(\Theta_{1}\right)^{2} .
\end{aligned}
$$

These transformations form a group which is called superconformal, or $\mathbf{C O N}_{1}$ in our notation. This group is well-studied in connection with the theory of the superconformal manifolds and is the main tool in constructing two-dimensional superconformal field theories [11], [12]. One can solve the equations (1.10) and so find finite superconformal transformations. However, the calculation uses anticommutation relations between $\theta$ and other thetas and it is not easy to generalize to arbitrary $p$. Anyway, it is much easier to work with infinitesimal transformations even in the Grassmann case.

For the infinitesimal form of the mapping (1.6), (1.7),

$$
\begin{aligned}
& \tilde{z}=z+\lambda \Omega(z, \theta)=z+\lambda\left(\omega_{0}+\theta \omega_{1}\right), \\
& \tilde{\theta}=\theta+\lambda \mathcal{E}(z, \theta)=\theta+\lambda\left(\epsilon_{0}+\theta \epsilon_{1}\right),
\end{aligned}
$$

the conditions (1.10) read

$$
\omega_{1}=\epsilon_{0} \quad, \quad \omega_{0}^{\prime}=2 \epsilon_{1} .
$$

Thus, defining the generators $\mathcal{T}\left(\omega_{0}\right)$ and $\mathcal{G}\left(\epsilon_{0}\right)$ by

$$
\tilde{F}=\left(1+\lambda\left(\mathcal{T}\left(\omega_{0}\right)+\mathcal{G}\left(\epsilon_{0}\right)\right)\right) F
$$

one can easily find that

$$
\mathcal{T}(\omega)=\omega \partial_{z}+\frac{1}{2} \omega^{\prime} \theta \partial_{\theta}, \quad \mathcal{G}(\epsilon)=\epsilon\left(\partial_{\theta}-\theta \partial_{z}\right)
$$

These generators close into the well-known Lie algebra

$$
\begin{aligned}
& {[\mathcal{T}(\omega), \mathcal{T}(\psi)]=\mathcal{T}\left(\omega \psi^{\prime}-\omega^{\prime} \psi\right)} \\
& {[\mathcal{T}(\omega), \mathcal{G}(\epsilon)]=\mathcal{G}\left(\omega \epsilon^{\prime}-\frac{1}{2} \omega^{\prime} \epsilon\right),} \\
& {[\mathcal{G}(\epsilon), \mathcal{G}(\zeta)]=\mathcal{T}(\epsilon \zeta) \text {. }}
\end{aligned}
$$

that we denote by $\mathcal{C O} \mathcal{N}_{1}$.

As in the previous example, it is possible to introduce similar generators (1.14) for the superconformal field $F$ of arbitrary weight $\Delta: F(z, \theta) \mapsto(\mathcal{D} \tilde{\theta})^{\Delta} F(\tilde{z}, \tilde{\theta})$ (cf. with (1.5)). The corresponding generators,

$$
\mathcal{T}(\omega)=\omega \partial_{z}+\frac{1}{2} \omega^{\prime} \theta \partial_{\theta}+\frac{\Delta}{2} \omega^{\prime}, \quad \mathcal{G}(\epsilon)=\epsilon\left(\partial_{\theta}-\theta \partial_{z}\right)-\Delta \epsilon^{\prime} \theta
$$

obey the same algebra (1.15). The operator $Q=\left(\partial_{\theta}-\theta \partial_{z}\right)$ which appeared in the definition of $\mathcal{G}(\epsilon)$ anticommutes with $\mathcal{D}$ and is called the supersymmetry generator. 
Up to now, $\omega$ and $\epsilon$ were respectively even and odd functions of the variables $z$ and of 'other thetas', i.e. some polynomials in 'other thetas' with coefficients being analytic functions of $z$. However, all $\theta$ variables anticommute $]^{2}$ and so we can move all parameters containing 'other thetas' to left-hand sides of all equations. This obviously allows to introduce 'bare' generators $T$ and $G$ whose arguments $\omega$ and $\epsilon$ are ordinary functions of $z$ independent of 'other thetas'. Then the expressions for $T$ and $G$ coincide with (1.9) and obey the algebra (1.15) with the last commutator replaced by anticommutator. This algebra (or, more precisely, the superalgebra) is what is usually called the 'superconformal algebra'. We will denote it by Con $_{1}$ and its unique central extension, the Ramond-Neveu-Schwarz algebra, is denoted by Vir .

The distinction between the algebras $\mathcal{C O N}_{1}$ and $\mathrm{Con}_{1}$ is usually ignored, being practically trivial. As we shall see in Sect.3, this is not the case for their paraanalogs $\mathcal{C O N}_{p}$ and $\mathrm{Con}_{p}$ since the paragrassmann algebra of 'other thetas' is a much more complicated object than the Grassmann one (and, probably, not uniquely defined, see [13]). In particular, we do not know at the moment simple and general commutation rules between the elements of the paragrassmann algebra. As a result, closing the algebra $\mathcal{C O} \mathcal{N}_{p}$ becomes a rather non-trivial problem. On the contrary, the algebra $\mathrm{Con}_{p}$ closes quite easily, even in several non-equivalent variants, if the number $p+1$ is rich in divisors. Each variant of closing defines an extended algebra, $V i r_{p}$, with a number of central charges (from 1 to $\left.[(p+1) / 2]\right)$.

The simplest variant of $V i r_{p}$ looks as follows:

$$
\begin{aligned}
{\left[L_{n}, L_{m}\right] } & =(n-m) L_{n+m}+\frac{2}{p+1}\left(\sum_{j} c_{j}\right)\left(n^{3}-n\right) \delta_{n+m, 0}, \\
{\left[L_{n}, G_{r}\right] } & =\left(\frac{n}{p+1}-r\right) G_{n+r} \\
\left\{G_{r_{0}}, \ldots, G_{r_{p}}\right\}_{c} & =(p+1) L_{\Sigma r}-\sum_{j} c_{j}\left(\sum_{i} r_{i} r_{i+j}+\frac{1}{p+1}\right) \delta_{\Sigma r, 0}, \\
j & =1 \ldots\left[\frac{p+1}{2}\right]
\end{aligned}
$$

where $L_{n}=T\left(z^{-n+1}\right), G_{r}=G\left(z^{-r+1 /(p+1)}\right)$, and $\{\ldots\}_{c}$ is the cyclic sum of the $(p+1)$-linear monomials:

$$
\left\{G_{0}, \ldots, G_{p}\right\}_{c}=G_{0} \cdots G_{p}+G_{p} \cdot G_{0} \cdots G_{p-1}+\ldots+G_{1} \cdots G_{p} \cdot G_{0} .
$$

Note that a particular variant of this algebra $V i r_{p}$, with totally symmetric bracket in the third line and without central extensions, had been presented in Ref.[14] under the name 'fractional Virasoro algebra'. Recently, a generalization that relates the algebras of Refs. [5] and [14], has been given in Ref.[7].

The rest of the paper is devoted to a generalization of the previous scheme to arbitrary integer $p$. In Sect.2, a necessary preliminary technique of the paragrassmann

\footnotetext{
${ }^{2}$ Note that the commutation relations between $\omega, \epsilon$, and $\theta$ are completely fixed by Eq. (1.9) and by the condition $\tilde{\theta}^{2}=0$.
} 
algebras is summarized and its versions most useful for constructing paraconformal transformations are presented. A detailed description of the differential calculus with one and many variables is given in Ref.[13].

In Sect.3, we first discuss main properties of the fractional derivative $\mathcal{D}\left(\mathcal{D}^{p+1}=\right.$ $\partial_{z}$ ) which are valid in any version of the paragrassmann calculus. Then, in the spirit of the above scheme, we introduce paraconformal transformations to construct a paraconformal group $\mathbf{C O N}_{p}$ and corresponding algebras $\mathcal{C O} \mathcal{N}_{p}$, and $\operatorname{Con}_{p}$ (Sect.4). We show that a $p$-analog of the infinitesimal transformations (1.11) must look as

$$
\begin{aligned}
& \tilde{\theta}=\theta+\lambda \mathcal{E}(z, \theta), \\
& \tilde{z}=z+\lambda \Omega^{(1)}+\ldots+\lambda^{p} \Omega^{(p)} .
\end{aligned}
$$

Unlike the Lie algebras (and superalgebras) having only first order generators, here we have to retain 'higher-order generators' thus introducing into consideration a $p$ jet structure. This suggests that the algebra $\mathcal{C O N}$ might be a $p$-filtered Lie algebra containing the generators of $p$ 'generations', $\left\{\mathcal{L}^{(i)}\right\}$, so that an analog of the formula (1.13) would look like

$$
\tilde{F}=\left(1+\lambda\left\{\mathcal{L}^{(1)}\right\}+\lambda^{2}\left\{\mathcal{L}^{(2)}\right\}+\ldots+\lambda^{p}\left\{\mathcal{L}^{(p)}\right\}\right) F .
$$

The algebra $\mathcal{C O N}_{p}$ contains generators of a new type (we call them $\mathcal{H}$-generators) that do not act on $z$ but are crucial in closing the algebra. The algebras $\operatorname{Con}_{p}$ can be closed without them.

In Sect.5, we briefly discuss the meaning of the construction in terms of algebraic geometry. This allows us to introduce central charges in a straightforward way and so to derive the algebras $V i r_{p}$. A discussion of the properties of these algebras, of possible generalizations, and of unsolved problems is given in Sect.6.

Appendix presents an explicit formulas for some paraconformal transformations for $p=2$ generalizing corresponding superconformal transformations. These formulas make more clear the analogies and differences between geometry of the superplane and that of the para-superplane.

Concluding this rather long introduction we would like to point out that the main formal results of this paper were known to us for some time 3 and have been presented at seminars and workshops this spring. However, we refrained from publishing them prior to understanding their geometric meaning. We hope that we can now suggest a possible geometric foundation for our formal construction in terms of the versions ('version covariance') and of jet-like structures although much remains to be done to completely uncover a geometric meaning of paraconformal transformations.

\section{Paragrassmann Algebra $\Pi_{p+1}$}

In Ref. [8] we have considered paragrassmann algebras $\Gamma_{p+1}(N)$ with $N$ nilpotent variables $\theta_{n}, \theta_{n}^{p+1}=0, n=1, \ldots, N$. Some wider algebras $\Pi_{p+1}(N)$ generated by $\theta_{n}$

\footnotetext{
${ }^{3} \mathrm{~A}$ preliminary version of this paper appeared under the same title as the preprint JINR E5-92393, Dubna, 1992. The present text is somewhat rearranged, made more concise, and new results have been added to Sect. 3 and Appendix.
} 
and additional nilpotent generators $\partial_{n}$ have also been constructed. These additional generators served for defining a paragrassmann differentiation and paragrassmann calculus. The building block for this construction was the simplest algebra $\Pi_{p+1}(1)$. By applying a generalized Leibniz rule for differentiations in the paragrassmann algebra $\Gamma_{p+1}(N)$ we have found two distinct realizations for $\Pi_{p+1}(1)$ closely related to the $q$-deformed oscillators. We have mentioned in [8] that other realizations of the $\Pi_{p+1}(1)$ may be constructed. A complete list of these realizations (versions) and a fairly general approach to constructing algebras $\Pi_{p+1}(N)$ have been presented in Ref.[13]. Here we reproduce the results of this work that are essential for understanding the main body of the present paper. Especially important is the fact that, under certain conditions, all these realizations are equivalent and one may choose those which are most convenient for particular problems.

The algebra $\Pi_{p+1}(1)$ is generated by the nilpotent variables $\theta$ and $\partial$ satisfying the conditions

$$
\theta^{p+1}=0=\partial^{p+1}
$$

(it is implied, of course, that $\theta^{p} \neq 0$ and the same for $\partial$ ). Any version of the algebra $\Pi_{p+1}(1)$ is defined by the relation allowing to move $\partial$ to the right of $\theta$

$$
\partial \theta=b_{0}+b_{1} \theta \partial+b_{2} \theta^{2} \partial^{2}+\ldots+b_{p} \theta^{p} \partial^{p},
$$

where $b_{i}$ are complex numbers restricted by consistency of the conditions (2.1) and (2.2) and by further assumptions to be formulated below. With the aid of Eq. (2.2) any element of the algebra can be expressed in terms of the basis $\theta^{r} \partial^{s}$, i.e. in the normal-ordered form. This relation obviously preserves the natural grading in the associative algebra generated by $\theta$ and $\partial$ satisfying (2.1)

$$
\operatorname{deg}\left(\theta^{r_{1}} \partial^{s_{1}} \theta^{r_{2}} \partial^{s_{2}} \ldots \theta^{r_{k}} \partial^{s_{k}}\right)=\Sigma r_{i}-\Sigma s_{i},
$$

A useful alternative set of parameters, $\alpha_{k}$, also fixing the algebra may be defined

$$
\partial \theta^{k}=\alpha_{k} \theta^{k-1}+(\ldots) \partial
$$

where dots denote a polynomial in $\theta$ and $\partial$. This relation is a generalization of the commutation relation for the standard derivative operator, $\partial_{z} z^{k}=k z^{k}+z^{(k-1)} \partial_{z}$, and we may define the differentiation of powers of $\theta$ by analogy,

$$
\partial\left(\theta^{k}\right)=\alpha_{k} \theta^{k-1}, \alpha_{0} \equiv 0
$$

to be justified later.

By applying Eq. (2.2) to Eq. (2.4) one may derive the recurrent relations connecting these two sets of the parameters:

$$
\alpha_{k+1}=\sum_{i=0}^{k} b_{i} \frac{\left(\alpha_{k}\right) !}{\left(\alpha_{k-i}\right) !},
$$

where $\left(\alpha_{k}\right) ! \equiv \alpha_{1} \alpha_{2} \cdots \alpha_{k}$. These relations enable us to express $\alpha_{k}$ as a function of the numbers $b_{i}, 0 \leq i \leq k-1$. The inverse operation, deriving $b_{i}$ in terms of $\alpha_{k}$, is well-defined only if all $\alpha_{k} \neq 0$. 
The consistency condition mentioned above is that the parameters must be chosen so as to satisfy the identity

$$
0 \equiv \partial \theta^{p+1}
$$

Taking into account that the second term in Eq. (2.4) vanishes for $k=p+1$ we have $\alpha_{p+1}=0$, with no other restrictions on the parameters $\alpha_{k}$ with $k \leq p$. The corresponding restriction on $p+1$ parameters $b_{i}$ follows from Eq. (2.6),

$$
\alpha_{p+1}\left(b_{0}, \ldots, b_{p}\right) \equiv b_{0}+b_{1} \alpha_{p}+b_{2} \alpha_{p} \alpha_{p-1}+\ldots+b_{p} \alpha_{p} \alpha_{p-1} \cdots \alpha_{2} \alpha_{1}=0,
$$

where the parameters $\alpha_{i}$ are expressed in terms of $b_{i}$. Any admissible set $\{b\}$ determines an algebra $\Pi_{p+1}^{\{b\}}$ with the defining relations (2.1), (2.2). To each algebra $\Pi_{p+1}^{\{b\}}$ there corresponds a set $\{\alpha\}$. A priori, there are no restrictions on $\{\alpha\}$, but, if we wish to treat $\partial$ as a non-degenerate derivative with respect to $\theta$, it is reasonable to require, in addition to (2.7), that

$$
\text { all } \alpha_{k} \neq 0
$$

So let us call a set $\{b\}$ (and corresponding algebra $\Pi_{p+1}^{\{b\}}$ ) non-degenerate, if the condition (2.8) is fulfilled, and degenerate otherwise. As it was already mentioned, in the non-degenerate case the numbers $b_{i}$ are completely determined by the numbers $\alpha_{k}$, so we can use the symbol $\{\alpha\}$ as well as $\{b\}$. An interesting fact is that nontrivial algebras $\left(\alpha_{1} \neq 0\right)$ may be degenerate if and only if $p+1$ is a composite number [13]. We will see later that the arithmetic properties of $p+1$ are also important in constructing paraconformal transformations.

In general, different sets $\{b\}$ determine non-equivalent algebras $\Pi_{p+1}^{\{b\}}$ and, at first sight, the algebras corresponding to different sets $\{b\}$ look very dissimilar. However, this is not true for the non-degenerate ones. In fact, all non-degenerate algebras $\Pi_{p+1}^{\{b\}}$ are isomorphic to the associative algebra $\operatorname{Mat}(p+1)$ of the complex $(p+1) \times(p+1)$ matrices.

The isomorphism can be manifested by constructing an explicit exact ('fundamental') representation for $\Pi_{p+1}^{\{b\}}$. With this aim we treat $\theta$ and $\partial$ as creation and annihilation operators (in general, not Hermitian conjugate) and introduce the ladder of $p+1$ states $|k\rangle, k=0,1, \ldots, p$ defined by

$$
\partial|0\rangle=0,|k\rangle \sim \theta^{k}|0\rangle, \theta|k\rangle=\beta_{k+1}|k+1\rangle .
$$

Here $\beta$ 's are some non-zero numbers, reflecting the freedom of the basis choice. As $|p+1\rangle=0$, the linear shell of the vectors $|k\rangle$ is finite-dimensional and in the nondegenerate case, when all $\beta_{k} \neq 0 \quad(k=1, \ldots, p)$, its dimension is $p+1$.

Using (2.9) and (2.4) we find

$$
\partial|k\rangle=\left(\alpha_{k} / \beta_{k}\right)|k-1\rangle
$$

Thus the fundamental (Fock-space) representations of the operators $\theta$ and $\partial$ is

$$
\begin{aligned}
& \theta_{m n}=\langle m|\theta| n\rangle=\beta_{n+1} \delta_{m, n+1}, \\
& \partial_{m n}=\langle m|\partial| n\rangle=\left(\alpha_{n} / \beta_{n}\right) \delta_{m, n-1} .
\end{aligned}
$$


It is not hard to see that for non-zero $\alpha$ 's, the matrices corresponding to $\theta^{m} \partial^{n} \quad(m, n=$ $0 \ldots p)$, form a complete basis of the algebra $\operatorname{Mat}(p+1)$. The isomorphism is established.

Thus, different non-degenerate algebras $\Pi_{p+1}^{\{b\}}$ are nothing more than alternative ways of writing one and the same algebra $\Pi_{p+1}$. We are calling them versions having in mind that fixing the $b$-parameters is somewhat analogous to a gauge-fixing (by adopting a broad meaning of this term introduced by $\mathrm{H}$. Weyl in his famous book on quantum mechanics).

This implies that we will mainly be interested in 'version-covariant' results, i.e. independent of a version choice. Nevertheless, special versions may have certain nice individual features making them more convenient for concrete calculations (thus allowing for simpler derivations of covariant results by non-covariant methods).

The same is true about the matrix representations. In principle, we need not use any matrix representation for the paragrassmann variables and derivatives. However, in some calculations the existence of the exact matrix representation (2.11), (2.12) is very useful for deriving version-covariant identities in the algebra $\Pi_{p+1}$. For instance, using the representation it is easy to check that

$$
\begin{aligned}
\left\{\partial, \theta^{(p)}\right\} & =\left(\sum \alpha_{k}\right) \theta^{p-1}, \\
\left\{\partial^{p}, \theta^{(p)}\right\} & =\prod \alpha_{k},
\end{aligned}
$$

and to find many other relations. Here we have introduced the notation

$$
\left\{\Xi, \quad \Psi^{(l)}\right\}=\Xi \Psi^{l}+\Psi \Xi \Psi^{l-1}+\ldots+\Psi^{l} \Xi
$$

that will be often used below. The identities (2.13) generalize those known in the para-supersymmetric quantum mechanics [4].

Note also that one may adjust the parameters $\beta_{k}$ to get a convenient matrix representation for $\theta$ and $\partial$. As a rule, we take $\beta_{k}=1$. For the versions with real parameters $\alpha_{k}$, it is possible to choose $\beta_{k}$ so as to have $\theta^{\dagger}=\partial$. We also normalize $\theta$ and $\partial$ so that $\alpha_{1} \equiv b_{0}=1$.

Now consider three special versions that are most suitable for constructing paraconformal transformations and correspond to simplest forms of Eq. (2.2).

(1): q-Version or Fractional Version

Here $b_{1}=q \neq 0, b_{2}=b_{3}=\ldots=b_{p}=0$, so that

$$
\alpha_{i}=1+q+\ldots+q^{i-1}=\frac{1-q^{i}}{1-q}
$$

The condition $\alpha_{p+1}=0$ tells that $q^{p+1}=1 \quad(q \neq 1)$, while the assumption that all $\alpha_{i} \neq 0$ forces $q=b_{1}$ to be a primitive root, i.e. $q^{n+1} \neq 1, n<p$. Thus, in this version $\left(\partial=\partial_{(1)}\right)$

$$
\begin{gathered}
\partial_{(1)} \theta=1+q \theta \partial_{(1)}, \\
\partial_{(1)}\left(\theta^{n}\right)=(n)_{q} \theta^{n-1}, \quad(n)_{q}=\frac{1-q^{n}}{1-q} .
\end{gathered}
$$


These relations were derived in Ref. [8] by assuming that $\partial$ is a generalized differentiation operator, i.e. satisfying a generalized Leibniz rule (a further generalization is introduced below). The derivative $\partial_{(1)}$ is naturally related to the $q$-oscillators (q-derivative) and to quantum algebras; Eq. (2.15) is also most convenient for generalizing to Paragrassmann algebras with many $\theta$ and $\partial$ (see [8], [13] and references therein).

(2): Almost Bosonic Version

For this Version

$$
b_{1}=1, \quad b_{2}=\ldots=b_{p-1}=0, \quad b_{p} \neq 0, \text { so that } \alpha_{k}=k
$$

The condition $\alpha_{p+1}=0$ gives $b_{p}=-\frac{p+1}{p !}$ and thus

$$
\left(\partial_{(2)}\right)_{m n}=n \delta_{m, n-1}, \quad \partial_{(2)} \theta=1+\theta \partial_{(2)}-\frac{p+1}{p !} \theta^{p} \partial_{(2)}^{p} .
$$

This derivative is 'almost bosonic' as $\partial_{(2)}\left(\theta^{n}\right)=n \theta^{n-1} \quad(n \neq p+1)$. This version is convenient for rewriting the generators of para-extensions of the Virasoro algebra in the most concise form in the next section.

Let us now discuss the interrelations between $\theta$ and $\partial$. As we have already mentioned the notation itself hints at treating $\partial$ as a derivative with respect to $\theta$ (see (2.4). To be more precise, let us represent an arbitrary vector $|F\rangle=\sum_{k=0}^{p} f_{k}|k\rangle$ as the function of $\theta$

$$
F(\theta)=\sum_{k=0}^{p} f_{k} \theta^{k} .
$$

The action of the derivative $\partial$ on $F(\theta)$ is defined by $(2.9)$ and 2.10$)\left(\beta_{k}=1\right)$,

$$
\partial(1)=0, \partial\left(\theta^{n}\right)=\alpha_{n} \theta^{n-1} \quad(1 \leq n \leq p) .
$$

It is clear, however, that this derivative does not obey the standard Leibniz rule $\partial(a b)=\partial(a) b+a \partial(b)$.

So consider the following modification of the Leibniz rule [8], [15]

$$
\partial(F G)=\partial(F) \bar{g}(G)+g(F) \partial(G) .
$$

The associativity condition (for differentiating $F G H$ ) tells that $g$ and $\bar{g}$ are homomorphisms, i.e.

$$
g(F G)=g(F) g(G), \quad \bar{g}(F G)=\bar{g}(F) \bar{g}(G) .
$$

The simplest natural homomorphisms compatible with the relations (2.17), (2.18), and (2.19) are linear automorphisms of the algebra $\Gamma_{p+1}$,

$$
g(\theta)=\gamma \theta, \quad \bar{g}(\theta)=\bar{\gamma} \theta
$$

where $\gamma, \bar{\gamma}$ are arbitrary complex parameters and

$$
\alpha_{k}=\frac{\bar{\gamma}^{k}-\gamma^{k}}{\bar{\gamma}-\gamma} .
$$


Using the condition (2.7) and assuming nondegeneracy, $\alpha_{n} \neq 0(n<p+1)$, we conclude that $\bar{\gamma} / \gamma$ must be a primitive $(p+1)$-root of unity. Thus we may formulate another interesting version of the paragrassmann algebra $\Pi_{p+1}$

\section{(3): $g-\bar{g}$-Version}

In this version the parameters $\alpha_{k}$ are given by Eq. (2.21), and we can calculate $b_{i}$ by solving Eq. (2.6): $b_{0}=1, b_{1}=\bar{\gamma}+\gamma-1, b_{2}=(\bar{\gamma}-\bar{\gamma} \gamma+\gamma-1) /(\bar{\gamma}+\gamma), \ldots$ Here $\gamma$ and $\bar{\gamma}$ are complex numbers constrained by the condition that $q=\bar{\gamma} / \gamma$ is a primitive root of unity. From Eqs. (2.18) and (2.20) one can derive the following operator relations for the automorphisms $g, \bar{g}$

$$
\partial \theta-\gamma \theta \partial=\bar{g}, \quad \partial \theta-\bar{\gamma} \theta \partial=g
$$

For the special case $\gamma=(\bar{\gamma})^{-1}=q^{1 / 2}$ identifying $\partial=a, \theta=a^{\dagger}$ allows to recognize in (2.22) the definitions of the $q$-deformed oscillators in the BiedenharnMacFarlane form [16]. Version-(1) can be derived from Version-(3) by putting $\bar{\gamma}=$ $q, \gamma=1$ (or $\bar{\gamma}=1, \gamma=q$ ). For $p=2$ all the above versions satisfy the modified Leibniz rule (2.18). However, for $p>2$ Version-(2) satisfies a different modification of the Leibniz rule

$$
\partial(F G)=\partial(F) \overline{\mathbf{g}}(G)+\mathbf{g}(F) \partial(G)+L z(F, G)
$$

For Version-(2) $\overline{\mathbf{g}}=\mathbf{g}=1$, and the additional term $L z(.,$.$) belongs to the one$ dimensional space $\{|p\rangle\}$. We suggest to call this term the 'Leibnizean'.

This modification as well as other versions are discussed in our accompanying paper [13]. There we also have constructed paragrassmann calculus with many variables. Note that a reasonably simple many-variable paragrassmann calculus with a generalized Leibniz rule can be formulated only for Versions (1) and (3). Nevertheless, Version-(2) is also useful as will be demonstrated below.

Before we proceed it is important to realize the following. While the Grassmann calculus satisfying our requirements is uniquef it is probably not true for many paragrassmann variables. We have proved that all nondegenerate algebras $\Pi_{p+1}(1)$ are equivalent but we have no corresponding theorem for the algebras $P(N)$ constructed in Ref.[13], and it is also possible that our construction does not exhaust many-variable algebras. For these reasons, in what follows we try to avoid, as much as possible, using any particular many-variable calculus. Then at some points we have to do some guesswork instead of precise computations and this results in some gaps in our geometric reasoning. Being fully aware of this, we still think that the geometric approach is indispensable for understanding paraconformal algebras, and so present it in its incomplete form. We hope that the gaps can be filled later as far as the many-variable paragrassmann calculus will be fully developed. Note also that the multilinear algebra $V i r_{p}$ though not precisely derived from the algebra $\operatorname{Con}_{p}$ has a self-dependent interest. With these cautionary remarks, we turn to our main task.

\footnotetext{
${ }^{4}$ For example, commuting Grassmann variables used in the Green ansatz are forbidden by the requirement that any linear combination of the Grassmann variables must also be a Grassmann variable, i.e. nilpotent.
} 


\section{Paraconformal Transformations}

To start realizing the program outlined in Introduction consider a para-superplane $\mathbf{z}=(z, \theta)$, where $z \in \mathbf{C}$ and $\theta$ is the generator of the paragrassmann algebra $\Gamma_{p+1}(1)=\Gamma_{\theta}$, i.e. $\theta^{p+1}=0$. Any function defined on this plane has the form

$$
F \equiv F(z, \theta)=F_{0}(z)+\theta F_{1}(z)+\theta^{2} F_{2}(z)+\ldots+\theta^{p} F_{p}(z) .
$$

It is useful to define an analog of the superderivative as a $(p+1)$-root of the derivative $\partial_{z}[8],[9]$

$$
\mathcal{D}=\partial_{\theta}+\kappa \frac{\theta^{p}}{\left(\alpha_{p}\right) !} \partial_{z}, \quad \mathcal{D}^{p+1}=\kappa \partial_{z} .
$$

We denote here the $\theta$-derivative in arbitrary version by $\partial_{\theta}$ instead of $\partial$ and shall often use this notation to make some formulas more transparent. The number $\kappa$ will be fixed later.

The action of this operator on the function (3.1) is

$$
\mathcal{D} F(z, \theta)=F_{1}(z)+\alpha_{2} \theta F_{2}(z)+\ldots+\alpha_{p} \theta^{p-1} F_{p}(z)+\kappa \frac{\theta^{p}}{\left(\alpha_{p}\right) !} F_{0}^{\prime}(z),
$$

where $F^{\prime}=\partial_{z} F$. In analogy with the super-calculus [11], [12] the inverse operator $\mathcal{D}^{-1}$ (defined up to a constant independent of $z$ and $\theta$ )

$$
\mathcal{D}^{-1} F \equiv \int^{\mathbf{z}} d \mathbf{z} F=\frac{\left(\alpha_{p}\right) !}{\kappa} \int^{z} d z^{\prime} F_{p}\left(z^{\prime}\right)+\theta F_{0}(z)+\frac{\theta^{2}}{\alpha_{2}} F_{1}(z)+\ldots+\frac{\theta^{p}}{\alpha_{p}} F_{p-1}(z)
$$

may be formally interpreted as an 'indefinite' integral.

Now we can formally introduce a 'definite' integral p

$$
\int_{\mathbf{z}_{2}}^{\mathbf{z}_{1}} d \mathbf{z} F=\int^{\mathbf{z}_{1}} d \mathbf{z} F-\int^{\mathbf{z}_{2}} d \mathbf{z} F .
$$

Of course, to make this definition and formulas below completely meaningful we have to use a well-defined paragrassmann calculus for many variables [ 0 . We instead are using some natural formal rules which have to be valid in different versions of the many-variable calculus. We assume that the action of the derivative $\partial_{\theta}$ on the expression having $\theta$ on the extreme left can be calculated by Eq.(2.4) and that the derivative of any expression containing no $\theta$-variable is zero (this is a nontrivial assumption as one can find considering examples of many-variable calculus [8], [13]). We will also as long as possible avoid assumptions on commuting different paragrassmann variables.

\footnotetext{
${ }^{5}$ The integral over $z$ is to be understood as a contour integral in the complex $z$-plane, and it is contour-independent as far as the contours are not crossing singularities of $F_{p}(z)$. Thus the integral might be regarded as a 'contour' integral in a para-superplane. If there is no singularity inside a closed contour, the integral must be zero.

${ }^{6}$ In fact we need an embedding of the algebra $\Gamma_{p+1}(1)$ into some infinite dimensional paragrassmann algebra $\Gamma_{p+1}(1+\infty)$ with generators $\theta_{0}=\theta, \theta_{1}, \theta_{2}, \ldots$.
} 
Now we can construct an analog of the Taylor expansion. Successively using the relation

$$
F\left(\mathbf{z}_{1}\right)-F\left(\mathbf{z}_{2}\right)=\int_{\mathbf{z}_{2}}^{\mathbf{z}_{1}} d \mathbf{z} \mathcal{D} F(\mathbf{z})
$$

and the identity

$$
\mathcal{D}^{n} F(\mathbf{z})=\mathcal{D}^{n} F\left(\mathbf{z}_{2}\right)+\left[\mathcal{D}^{n} F(\mathbf{z})-\mathcal{D}^{n} F\left(\mathbf{z}_{2}\right)\right]
$$

one can write a generalized Taylor expansion in the form

$$
F\left(\mathbf{z}_{1}\right)=\sum_{n} I_{n}\left(\mathbf{z}_{1}, \mathbf{z}_{2}\right) \mathcal{D}^{n} F\left(\mathbf{z}_{2}\right)
$$

where $I_{0}=1$ and

$$
I_{n+1}\left(\mathbf{z}_{1}, \mathbf{z}_{2}\right)=\int_{\mathbf{z}_{2}}^{\mathbf{z}_{1}} d \mathbf{z} I_{n}\left(\mathbf{z}, \mathbf{z}_{2}\right) .
$$

The first $p$ integrals are easy to compute:

$$
\begin{gathered}
I_{1}=\theta_{1}-\theta_{2} \\
I_{2}=\frac{1}{\alpha_{2}} \theta_{1}^{2}-\theta_{1} \theta_{2}+\left(1-\frac{1}{\alpha_{2}}\right) \theta_{2}^{2}, \ldots \\
I_{p+1}=\frac{1}{\kappa}\left(z_{1}-z_{2}\right)-\frac{1}{\left(\alpha_{p}\right) !} \theta_{1}^{p} \theta_{2}+\left(1-\frac{1}{\alpha_{2}}\right) \frac{1}{\left(\alpha_{p-1}\right) !} \theta_{1}^{p-1} \theta_{2}^{2}+\ldots .
\end{gathered}
$$

In view of Eq.(3.2) and by analogy with the Grassmann case [11], [12] one might expect that all the higher integrals can be expressed in terms of the first $p+1$ integrals and that these integrals are translation invariant. This is not quite the case for arbitrary $p$, the generalization is not so naive and requires some care. For not to digress from our main goal we postpone a detailed treatment of the integrals to a separate publication. The simplest case $p=2$ is briefly outlined in Appendix after introducing a generalization of super-translations, which is also a nontrivial problem.

We think that these properties of $\mathcal{D}$ and $\mathcal{D}^{-1}$ justify regarding $\mathcal{D}$ as a correct generalization of the superderivative and we proceed with realizing our program. Thus consider invertible transformations of the para-superplane

$$
\begin{gathered}
z \rightarrow \tilde{z}(z, \theta), \quad \operatorname{deg}(\tilde{z})=0, \\
\theta \rightarrow \tilde{\theta}(z, \theta), \quad \operatorname{deg}(\tilde{\theta})=1 ; \\
\tilde{z}=Z_{0}(z)+\theta Z_{1}(z)+\ldots+\theta^{p} Z_{p}(z), \\
\tilde{\theta}=\Theta_{0}(z)+\theta \Theta_{1}(z)+\ldots+\theta^{p} \Theta_{p}(z),
\end{gathered}
$$

where deg is a natural $\mathbf{Z}_{p+1}$-grading in $\Gamma_{p+1}(1+\infty)$ and $Z_{i}$ and $\Theta_{i}$ are functions of $z$ with values in $\Gamma_{p+1}(1+\infty) / \Gamma_{\theta}$ of needed grading. Here we assume that it is possible to move all $\theta$ to the left-hand sides of the para-superfields (3.8) and (3.9) (for Version-(1) it is evident). We also require that

$$
\tilde{\theta}^{p+1}=0
$$


and, of course, $[\tilde{z}, \tilde{\theta}]=0$.

The corresponding transformation for the functions (3.1) is defined as

$$
\tilde{F} \equiv \tilde{F}(z, \theta)=F(\tilde{z}, \tilde{\theta})=F_{0}(\tilde{z})+\tilde{\theta} F_{1}(\tilde{z})+\ldots+\tilde{\theta}^{p} F_{p}(\tilde{z}) .
$$

In accord with $(3.3), \tilde{\mathcal{D}} \tilde{F}$ is

$$
\tilde{\mathcal{D}} \tilde{F}=F_{1}(\tilde{z})+\tilde{\theta} F_{2}(\tilde{z})+\ldots+\frac{\kappa}{\left(\alpha_{p}\right) !} \tilde{\theta}^{p} F_{0}^{\prime}(\tilde{z}) .
$$

Following the route described in Section 1, consider the transformations obeying the requirement analogous to Eq. (1.9)

$$
\mathcal{D} F(\tilde{\theta}, \tilde{z})=\Phi(\theta, z) \tilde{\mathcal{D}} F(\tilde{\theta}, \tilde{z})
$$

or, in the operator form,

$$
\mathcal{D}=\Phi \tilde{\mathcal{D}}
$$

Acting on $\tilde{\theta}$ we immediately get

$$
\Phi=\mathcal{D} \tilde{\theta}
$$

Consider main properties of these transformations. They satisfy the group property since for two sequential transformations $\mathbf{z} \mapsto \tilde{\mathbf{z}}(\mathbf{z}) \mapsto \tilde{\tilde{\mathbf{z}}}(\tilde{\mathbf{z}}(\mathbf{z})$ ) we have (see (3.14), (3.15))

$$
\mathcal{D}=(\mathcal{D} \tilde{\theta}) \tilde{\mathcal{D}}=(\mathcal{D} \tilde{\theta})(\tilde{\mathcal{D}} \tilde{\tilde{\theta}}) \tilde{\tilde{\mathcal{D}}}=(\mathcal{D} \tilde{\tilde{\theta}}) \tilde{\tilde{\mathcal{D}}}
$$

This group will be called 'paraconformal' and referred to as $\mathbf{C O N}_{p}$.

The condition (3.14) is a very strong restriction on possible form of transformation functions (3.7). One can show that all the restrictions can be derived by putting $F$ to be $\tilde{\theta}^{k}, \tilde{z}$ and $\tilde{\theta} \tilde{z}$. This gives

$$
\begin{aligned}
\mathcal{D} \tilde{\theta}^{k} & =\alpha_{k}(\mathcal{D} \tilde{\theta}) \tilde{\theta}^{k-1}, \\
\mathcal{D} \tilde{z} & =\frac{\kappa}{\left(\alpha_{p}\right) !}(\mathcal{D} \tilde{\theta}) \tilde{\theta}^{p}, \\
\mathcal{D}(\tilde{\theta} \tilde{z}) & =(\mathcal{D} \tilde{\theta}) \tilde{z} .
\end{aligned}
$$

Note that (3.18) allows us to interpret (3.14) as the rule for differentiation of composite functions

$$
\mathcal{D}=(\mathcal{D} \tilde{\theta})\left(\tilde{\partial}_{\theta}+\kappa \frac{\tilde{\theta}^{p}}{\left(\alpha_{p}\right) !} \tilde{\partial}_{z}\right)=(\mathcal{D} \tilde{\theta}) \tilde{\partial}_{\theta}+(\mathcal{D} \tilde{z}) \tilde{\partial}_{z}
$$

Summarizing the main properties of the derivative $\mathcal{D}$ and of the transformations satisfying (3.14), we conclude that it is reasonable to regard them as the paraconformal transformations of the para-superplane.

The main restrictions on the parameters of paraconformal transformations are coming from Eq. (3.18) \ that, taken in components, give rise to the following

\footnotetext{
${ }^{7}$ Eqs.(3.17), (3.19) give additional restriction, mostly on the commutation properties of the parameters.
} 
relations between $Z_{i}$ and $\Theta_{j}$ (defined by (3.8), (3.9))

$$
\begin{aligned}
Z_{1} & =\frac{\kappa}{\left(\alpha_{p}\right) !} \Theta_{1} \Theta_{0}^{p}, \\
\theta Z_{2} & =\frac{\kappa}{\left(\alpha_{p}\right) !}\left(\theta \Theta_{2} \Theta_{0}^{p}+\frac{1}{\alpha_{2}} \Theta_{1}\left\{\theta \Theta_{1}, \Theta_{0}^{(p-1)}\right\}\right), \\
& \cdots \\
\theta^{p-1} Z_{p} & =\frac{\kappa}{\left(\alpha_{p}\right) !}\left(\theta^{p-1} \Theta_{p} \Theta_{0}^{p}+\ldots+\frac{1}{\alpha_{p}} \Theta_{1}\left\{\left(\theta \Theta_{1}\right)^{(p-1)}, \Theta_{0}\right\}\right), \\
\theta^{p} Z_{0}^{\prime} & =\frac{\kappa}{\left(\alpha_{p}\right) !} \theta^{p} \Theta_{0}^{\prime} \Theta_{0}^{p}+\alpha_{p} \theta^{p-1} \Theta_{p}\left\{\theta \Theta_{1}, \quad \Theta_{0}^{(p-1)}\right\}+\ldots+\Theta_{1} \cdot\left(\theta \Theta_{1}\right)^{p} .
\end{aligned}
$$

Here the notation introduced in (2.14) is used.

These relations generalize the much simpler relations (1.10) to any $p$. It is rather hard to push forward the analysis with complicated expressions (3.21) without having a well-established technique for handling many thetas. For this reason, we are forced to turn to the infinitesimal language. Then, introducing a small $c$-number parameter $\lambda$ one may rewrite the transformations (3.7-3.9) as

$$
\begin{aligned}
& \tilde{z}(z, \theta)=z+\lambda \Omega(z, \theta)+O\left(\lambda^{2}\right), \quad \Omega=\sum_{i=0}^{p} \theta^{i} \omega_{i}(z) \\
& \tilde{\theta}(z, \theta)=\theta+\lambda \mathcal{E}(z, \theta)+O\left(\lambda^{2}\right), \quad \mathcal{E}=\sum_{i=0}^{p} \theta^{i} \epsilon_{i}(z) .
\end{aligned}
$$

where $\omega_{i}$ and $\epsilon_{i}$ are first coefficients in expansions of the functions $Z_{i}(z)$ and $\Theta_{i}(z)$ in powers of $\lambda$. A priori, there is no reason to exclude the higher powers from consideration. The infinitesimal transformations (3.22), (3.23) satisfying the paraconformal conditions $(3.17-3.19)$ define a linear space which we denote by $\mathcal{C O N}_{p}$. This is an infinitesimal object corresponding to the paraconformal group $\mathbf{C O N}_{p}$ and so it must carry some algebraic structure induced by the group structure of $\mathbf{C O N}_{p}$. In this sense, we will speak of 'the algebra $\mathcal{C O N}_{p}$ ' though its algebraic properties will be discussed later. Here we briefly analyze a geometric meaning of $\mathcal{C O N}_{p}$.

As it is evident from (3.23), the only component of $\tilde{\theta}$ containing a finite part is $\Theta_{1}=1+\lambda \epsilon_{1}(z)$; the rest of $\Theta_{i}$ are of the first order in $\lambda$. Then (3.21) tells that only $Z_{0}$ and $Z_{p}$ contain the terms of the first order in $\lambda$, while the other $Z_{i}$ with $1 \leq i \leq p-1$ must be of the order $\lambda^{p+1-i}$. This suggests that all terms up to the order $\lambda^{p}$ must be kept in (3.22), (3.23). So, since the transformed function is generally defined as $\tilde{F}=(1+($ general element of the algebra $)) F,($ cf. (1.13) $)$ a general element of the algebra $\mathcal{C O N}$ p must be of the form

$$
(\text { general element })=\lambda\left\{\mathcal{L}^{(1)}\right\}+\lambda^{2}\left\{\mathcal{L}^{(2)}\right\}+\ldots+\lambda^{p}\left\{\mathcal{L}^{(p)}\right\} .
$$

Here we denote by $\left\{\mathcal{L}^{(M)}\right\}$ a set of the generators of the $M$-th generation. Eq.(3.21) shows that $M$-th generation $\left\{\mathcal{L}^{(M)}\right\}$ must contain some new generators $\left\{\mathcal{X}^{(M)}\right\}$ that are not present in $\left\{\mathcal{L}^{(M-1)}\right\}$. This would guarantee, in particular, the appearance of non-zero $Z_{p+1-M}$. If it were possible to put all $Z_{i}(i=1 \ldots p-1)$ to zero we would get rid of these subtleties. But as we will see below, this contradicts to the requirement of the bilinear closure of the algebra $\mathcal{C O N}_{p}$. In fact, new generators naturally arise from certain bilinear brackets of the old ones, and this process stops only at the $p$-th generation. 
Returning to geometry, the formula (3.24) indicates that the algebra $\mathcal{C O N}_{p}$ lives not in the tangent space of the group but rather in a space of $p$-jets (see, e.g. Ref.[17]). For the $p$-jets the generators must be of the form (something) $\partial^{j} \quad(j=$ $1 \ldots p)$. This is right the case for the algebra $\mathcal{C O N}_{p}$, as we will see below?

Now an explicit realization of the paraconformal algebra generators $\mathcal{L}^{(M)}$ is in order. We concentrate on the first generation because generators of the higher generations must be obtained through multilinear combinations of them. Substituting (3.22) (3.23) in (3.17) - (3.19) we get the (first-order smallness) infinitesimal form of the paraconformal conditions:

$$
\begin{aligned}
\mathcal{D}\left\{\mathcal{E}, \theta^{(k)}\right\} & =(k+1)\left((\mathcal{D E}) \theta^{k}+\left\{\mathcal{E}, \theta^{(k-1)}\right\}\right), \quad k=1 \ldots p-1 \\
\mathcal{D} \Omega & =\frac{\kappa}{\left(\alpha_{p}\right) !}\left((\mathcal{D} \mathcal{E}) \theta^{p}+\left\{\mathcal{E}, \theta^{(p-1)}\right\}\right) \\
\mathcal{D}(\mathcal{E} z+\theta \Omega) & =(\mathcal{D} \mathcal{E}) z+\Omega
\end{aligned}
$$

Eqs. 3.25 - 3.27) lead to certain restrictions on the functions $\epsilon_{i}, \omega_{i}$ (we assume that $\omega_{0}$ and $\epsilon_{1}$ are ordinary functions, free of any paragrassmann content). The condition (3.26) gives

$$
\begin{aligned}
\omega_{1}=\omega_{2} & =\ldots=\omega_{p-1}=0, \\
\epsilon_{1} & =\frac{1}{p+1} \omega_{0}^{\prime}, \\
\alpha_{p} \theta^{p-1} \omega_{p} & =\frac{\kappa}{\left(\alpha_{p}\right) !}\left\{\epsilon_{0}, \theta^{(p-1)}\right\},
\end{aligned}
$$

wherefrom, by virtue of the relation $\left\{\epsilon_{0}, \theta^{(p)}\right\}=0$ that follows from the nilpotency condition (3.10), we have

$$
\theta^{p} \omega_{p}=-\frac{1}{\alpha_{p}} \frac{\kappa}{\left(\alpha_{p}\right) !} \epsilon_{0} \theta^{p}
$$

From the third relation (3.27) we find that

$$
\theta^{p} \omega_{p}=\frac{\kappa}{\left(\alpha_{p}\right) !} \theta^{p} \epsilon_{0}, \text { or } \omega_{p}=\frac{\kappa}{\left(\alpha_{p}\right) !} \epsilon_{0},
$$

which, together with (3.31), gives the commutation relation

$$
\epsilon_{0} \theta^{p}+\alpha_{p} \theta^{p} \epsilon_{0}=0
$$

The condition (3.25) provides the commutation rules of $\epsilon_{i}$ with $\theta^{k}$ and $\partial$ :

$$
\partial\left(\theta^{i}\left\{\epsilon_{i}, \theta^{(k)}\right\}\right)=(k+1)\left(\alpha_{i} \theta^{i-1} \epsilon_{i} \theta^{k}+\theta^{i}\left\{\epsilon_{i}, \theta^{(k-1)}\right\}\right), \quad k=1 \ldots p-1 .
$$

We emphasize that, in general, these rules do not require any $\epsilon_{i}$ to be zero.

\footnotetext{
${ }^{8}$ In geometric terms, one has to consider tangent bundles of the order $p$. There exist a natural relation between $\Gamma_{p+1}(1)$ and tangent bundles of the order $p$ studied in the context of A.Weil's bundles of infinitely near points (see, e.g. Ref.[18]). Although this relation might be useful also in our case, we apparently need to deal with more complex structures.
} 
Thus the resulting infinitesimal paraconformal transformations of the first order in $\lambda$ look as follows

$$
\begin{aligned}
\delta z & =\lambda\left(\omega_{0}(z)-\frac{1}{\alpha_{p}} \frac{1}{\left(\alpha_{p}\right) !} \epsilon_{0}(z) \theta^{p}\right), \\
\delta \theta & =\lambda\left(\epsilon_{0}(z)+\frac{1}{p+1} \omega_{0}^{\prime}(z) \theta+\theta^{2} \epsilon_{2}+\ldots+\theta^{p} \epsilon_{p}\right), \\
\delta\left(\theta^{k}\right) & =\lambda\left(\left\{\epsilon_{0}, \theta^{(k-1)}\right\}+\frac{k}{p+1} \omega_{0}^{\prime} \theta^{k}+\theta^{2}\left\{\epsilon_{2}, \theta^{(k-1)}\right\}+\ldots+\theta^{p+1-k}\left\{\epsilon_{p+1-k}, \theta^{(k-1)}\right\}\right) .
\end{aligned}
$$

To obtain generators of the transformations (3.35), it is convenient to define new operators $\mathcal{J}_{0}$ and $\bar{\partial}$ acting on $\theta^{k}$ in the following way

$$
\begin{aligned}
\mathcal{J}_{0}\left(\theta^{k}\right) & =k \theta^{k}, \\
\epsilon_{i} \bar{\partial}\left(\theta^{k}\right) & =\left\{\epsilon_{i}, \theta^{(k-1)}\right\}, .
\end{aligned}
$$

The first operator is a generator of the automorphism group of paragrassmann algebra [8]. The second one can be interpreted as differentiation in certain other version $(\bar{N})$, which is, in this sense, an 'associate' to the original version $(N)$. So, in addition to (3.37), we assume that there exist a set of non-zero numbers $\bar{\alpha}_{k}$ such that

$$
\bar{\partial}\left(\theta^{k}\right)=\bar{\alpha}_{k} \theta^{k-1}
$$

This assumption is not too strong but, together with (3.37), it leads to certain non-trivial restrictions on commutation of $\epsilon_{i}$ and $\theta$. We will not try to specify the associate version in general. For the moment it is sufficient to know that for Version- $(1)_{q}$ and Version-(2) the associate versions are $(1)_{q^{-1}}$ and Version (2) itself, respectively.

Now it is convenient to introduce the operator

$$
Q=\bar{\partial}-\frac{1}{\alpha_{p}} \frac{\kappa}{\left(\alpha_{p}\right) !} \theta^{p} \partial_{z}
$$

and choose $\kappa=-\alpha_{p} \frac{\left(\alpha_{p}\right) !}{\left(\bar{\alpha}_{p}\right) !}$, so that $Q^{p+1}=\partial_{z}(Q$ is an analog of the supersymmetry generator and might be called the para-supersymmetry generator).

By virtue of these operators we can establish the generators of the transformations (3.35) in any version (from now on we omit the zero index of $\omega_{0}$ )

$$
\begin{aligned}
T(\omega) & =\omega \partial_{z}+\frac{1}{p+1} \omega^{\prime} \mathcal{J}_{0}, \\
\mathcal{G}\left(\epsilon_{0}\right) & =\epsilon_{0}\left(\bar{\partial}_{\theta}+\frac{\theta^{p}}{\left(\bar{\alpha}_{p}\right) !} \partial_{z}\right)=\epsilon_{0} Q, \\
\mathcal{H}_{j}\left(\epsilon_{j+1}\right) & =\theta^{j+1} \epsilon_{j+1} \bar{\partial}_{\theta} .
\end{aligned}
$$

These are the generators of the first generation $\left\{\mathcal{L}^{(1)}\right\}$. They must generate, through correct bilinear and multilinear brackets, the entire algebra $\mathcal{C O} \mathcal{N}_{p}$ corresponding to the group $\mathbf{C O N}_{p}$. 
Let us now describe these generators in the preferred versions.

Version- $(1)_{q}$.

Remember that this means $\partial \theta=1+q \theta \partial$, and therefore $\partial \theta^{k}=(k)_{q} \theta^{k-1}+q^{k} \theta^{k} \partial$. Then, assuming the commutation relations $\epsilon_{i} \theta=r_{i} \theta \epsilon_{i}$, where $r_{i}$ are some numbers, we find from (3.34) that all these factors $r_{i}$ must be equal to $q$ (for $\epsilon_{0}$ this can be seen from (3.33) since $\left.\alpha_{p}=(p)_{q}=-q^{p}\right)$. Now recalling the definition of $\bar{\partial}$ (3.37) we find that

$$
\bar{\partial}\left(\theta^{k}\right)=(k)_{q^{-1}} \theta^{k-1} \equiv q^{1-k}(k)_{q} \theta^{k-1},
$$

and so $\bar{\partial}$ may be represented as $\bar{\partial}=g^{-1} \partial$, where $g^{-1}$ is an automorphism of the paragrassmann algebra $\Gamma_{\theta}$ defined by $g^{-1}\left(\theta^{k}\right)=q^{-k} \theta^{k}$. It is easy to prove (see [8]) that

$$
\begin{aligned}
g & =q^{\mathcal{J}_{0}}=\partial \theta-\theta \partial \\
g^{-1} & =q^{-\mathcal{J}_{0}}=\bar{\partial} \theta-\theta \bar{\partial}
\end{aligned}
$$

Thus, we see that $\bar{\partial}$ is in fact a differentiation in the associate version $(1)_{q^{-1}}$. The generators $\mathcal{G}$ and $\mathcal{H}$ are represented as

$$
\begin{aligned}
\mathcal{G}(\epsilon) & =\epsilon\left(\bar{\partial}_{\theta}+\frac{\theta^{p}}{(p)_{q^{-1}} !} \partial_{z}\right)=\epsilon Q, \\
\mathcal{H}_{j}\left(\epsilon_{j+1}\right) & =q^{-(j+1)} \epsilon_{j+1} \theta^{j+1} \bar{\partial}_{\theta} .
\end{aligned}
$$

while $T$-generator has the same form as in Eqs. (3.40).

We would like to remind that the operator $\mathcal{D}(3.2)$ in Version- $(1)_{q}$ and the parasupersymmetry generator $Q$ (3.41) have been introduced earlier in the context of the fractional supersymmetry [9].

Version-(2).

The unique property of this version is that the $\theta$-derivative acts exactly like the standard one except the terms proportional to $\theta^{p}$ in the Leibniz rule and corresponding terms in the operator formulas. This deviation from the standard rules is not important in considering infinitesimal paraconformal transformations (3.35).

The basic formula of Version-(2) required in this context is

$$
\partial \theta^{j}=j \theta^{j-1}+\theta^{j} \partial-\frac{p+1}{(p+1-j) !} \theta^{p} \partial^{p+1-j}, \quad j=1 \ldots p+1 .
$$

Applying it to the relations

$$
\partial\left(\left\{\epsilon_{0}, \theta^{(k)}\right\}\right)=(k+1)\left\{\epsilon_{0}, \theta^{(k-1)}\right\}, \quad k=1 \ldots p-1,
$$

which are just (3.34) for $\epsilon_{0}$, one can easily get by induction that

$$
\partial\left(e_{0} \theta^{k}\right)=k \epsilon_{0} \theta^{k-1}, \quad k=1 \ldots p-1 .
$$


On the other hand, remembering the definition of $\bar{\partial}(3.37$ - 3.38), we may regard (3.44) as the recurrence equation defining $\bar{\alpha}_{k}$

$$
\bar{\alpha}_{k+1} \partial\left(\epsilon_{0} \theta^{k}\right)=(k+1) \bar{\alpha}_{k} \epsilon_{0} \theta^{k-1}, \quad k=1 \ldots p-1 .
$$

On account of (3.45), we get $\bar{\alpha}_{k}=k$ for all $k=1 \ldots p$ since $\bar{\alpha}_{1}=1$. Therefore $\bar{\partial} \equiv \partial$ and the associate version is identical to Version-(2) itself.

This may be considered as a simplest example of a general procedure for obtaining the associate version. Note also that the relations (3.45) together with $\partial\left(e_{0} \theta^{p}\right)=-p^{2} \theta^{p-1} \epsilon_{0} \equiv-\frac{p}{(p-1) !} \theta^{p-1} \epsilon_{0} \partial^{p}\left(\theta^{p}\right)$ can be summarized in a single operator formula

$$
\partial \epsilon_{0}=\epsilon_{0} \partial-\frac{p}{(p-1) !} \theta^{p-1} \epsilon_{0} \partial^{p}-\frac{1}{(p-1) !} \epsilon_{0} \theta^{p-1} \partial^{p} .
$$

This is an example of how the commutation relations of an algebra with many paragrassmann variables can look in a version other than Version-(1). In general, they look monstrous and not suitable for computations.

The operator $\mathcal{J}_{0}$, standing in the $T$-generator, has a very simple expression in Version- $(2): \mathcal{J}_{0}=\theta \partial$, and so the generators (3.40) look in this version in a quite vector-like fashion

$$
\begin{aligned}
T(\omega) & =\omega \partial_{z}+\frac{1}{p+1} \omega^{\prime} \theta \partial_{\theta}, \\
\mathcal{G}\left(\epsilon_{0}\right) & =\epsilon_{0}\left(\partial_{\theta}+\frac{\theta^{p}}{p !} \partial_{z}\right), \\
\mathcal{H}_{j}\left(\epsilon_{j+1}\right) & =\theta^{j+1} \epsilon_{j+1} \partial_{\theta} .
\end{aligned}
$$

Usually, to derive some identities between generators, it is better to take them in Version-(2), in the form (3.47). Version-(1) is better adapted to the computations with many paragrassmann variables.

\section{Paraconformal Algebras}

Now let us turn to the algebra of the generators (3.40). The commutators with $T$ are simple as they should be due to the commutativity of $\omega$ :

$$
\begin{aligned}
{[T(\omega), T(\eta)] } & =T\left(\omega \eta^{\prime}-\omega^{\prime} \eta\right) \\
{[T(\omega), \mathcal{G}(\epsilon)] } & =\mathcal{G}\left(\omega \epsilon^{\prime}-\frac{1}{p+1} \omega^{\prime} \epsilon\right), \\
{\left[T(\omega), \mathcal{H}_{j}(\xi)\right] } & =\mathcal{H}_{j}\left(\omega \xi^{\prime}+\frac{j}{p+1} \omega^{\prime} \xi\right) .
\end{aligned}
$$

The rest of the commutators promise some subtleties. For instance, the commutator of two $\mathcal{G}$-generators gives rise to a new generator $\mathcal{G}^{(2)}$

$$
[\mathcal{G}(\epsilon), \mathcal{G}(\zeta)] \propto \mathcal{G}^{(2)}(\epsilon \zeta-\zeta \epsilon)+\frac{1}{2} \mathcal{H}_{p-1}\left(\epsilon \zeta^{\prime}-\epsilon^{\prime} \zeta+\zeta^{\prime} \epsilon-\zeta \epsilon^{\prime}\right)
$$


This new generator has the form

$$
\mathcal{G}^{(2)}(\phi)=\phi Q^{2}+\frac{1}{2} \phi^{\prime} \frac{\theta^{p}}{\left(\bar{\alpha}_{p}\right) !} \bar{\partial}
$$

and contains a term $\phi \theta^{p-1} \partial_{z}$, modifying $z$ on a quantity proportional to $\theta^{p-1}$, that is forbidden by the condition (3.28). Note, however, that (3.28) is a condition on the first-order variation, while $\mathcal{G}^{(2)}$ appears only in the second order. Similar effects occur when considering commutators of two $\mathcal{H}$-generators or of $\mathcal{G}$ and $\mathcal{H}$, giving rise to the generators $\mathcal{H}_{j}^{(2)}$. The latter have the form $\mathcal{H}_{j}^{(2)}(\psi) \propto \psi \theta^{j+1} \partial^{2}$.

$\mathcal{G}^{(2)}$ and $\mathcal{H}_{j}^{(2)}$ are right those new generators of the second generation, $\left\{\mathcal{X}^{2}\right\}$, expected to appear in the term of order $\lambda^{2}$ in (3.24). They can be obtained directly by repeating the steps 3.25 - 3.40) but with accounting $\lambda^{2}$ terms in (3.22), (3.23).

Similar procedure can be carried out (but the calculations become more and more complicated) for $\lambda^{M}, M=3,4$, etc. , giving rise to the generators of $M$-th generation $\left\{\mathcal{G}^{(M)}, \mathcal{H}_{j}^{(M)}\right\} \equiv\left\{\mathcal{X}^{M}\right\}$. Generators $\mathcal{G}^{(M)}$ are right those that contain a term $\sim \theta^{p+1-M} \partial_{z}$, which gives rise to non-zero $Z_{p+1-M}$ in (3.21). Generators $\mathcal{H}_{j}^{(M)}$ are proportional to $\theta^{j+1} \bar{\partial}^{M}$ and do not affect $z$-coordinate. They lead to a deviation of $\theta^{M}$ from $(\tilde{\theta})^{M}$ on a quantity of order $\lambda^{M}$. Probably, this could be interpreted as a shift of the version during a paraconformal transformation.

New generators stop appearing at the order $\lambda^{p}$. This fact could be explained by two circumstances. First: the resource of possible combinations of $\theta, \bar{\partial}$ and $\partial_{z}$, which are the building blocks for generators, is exhausted at the order $\lambda^{p}$. Second, (closely related to the first): the algebra of the generators

$$
\left\{\mathcal{L}^{p}\right\}=\left\{\mathcal{L}^{1}\right\} \cup\left\{\mathcal{X}^{2}\right\} \cup \ldots \cup\left\{\mathcal{X}^{p}\right\}=\left\{T, \mathcal{G}, \mathcal{H}_{j} ; \mathcal{G}^{2}, \mathcal{H}_{j}^{2} ; \ldots ; \mathcal{G}^{p}, \mathcal{H}_{j}^{p}\right\}
$$

closes bilinearly. So, if we denote by $A^{(M)}$ the linear shell of all the generators of $M$-th generation, $\left\{\mathcal{L}^{M}\right\}$, then the entire algebra $\mathcal{C O} \mathcal{N}_{p}$ can be represented as a $p$-filtered algebra, i.e. the generators can be ordered as follows

$$
\mathcal{C O N}_{p}=A^{(p)} \supset A^{(p-1)} \supset \ldots \supset A_{(2)} \supset A^{(1)} ;\left[A^{(M)}, A^{(K)}\right]_{M K} \subset A^{(M+K)} .
$$

Each coset $A^{(M)} / A^{(M-1)}$ is based on the generators $\mathcal{X}^{(M)}$.

Of course, this is a somewhat symbolic statement, because explicit expressions for the bilinear brackets $[\ldots]_{M K}$ are not known to us as yet, except the bracket $[\ldots]_{11}$, which is simply the commutator. In general, it is not clear which combination of the para-generators (this word applies to $\mathcal{G}$ - and $\mathcal{H}$-type ones in any generation) should be taken, because the true bracket should be determined by some analog of the Hausdorff formula for the para-supergroup, and the latter is not obtained so far. Though taking the commutator of two $\mathcal{G}$-generators seems to be more or less consistent with the first order calculation, the commutator of, say, $\mathcal{G}$ and $\mathcal{G}^{(2)}$ seems hardly to have any relation to the group $\mathbf{C O N}_{p}$. We would not like also to care about commutation properties of $\epsilon_{i}$, especially taking into account that for general elements of the paragrassmann algebra of many variables the commutation formulas are not specified. For $p=2$ we could do more as one may see from the 
explicit formulas presented in Appendix. We will give a detailed consideration of this simplest case in our next paper.

Just now we explore another approach, not restricted to special values of $p$ and to particular versions of many-variable algebras. Let us extract paragrassmann multipliers out of all $\epsilon$ and omit them, in analogy to what is done in the supercase, leaving the arguments of all generators to be just ordinary functions commuting with everything. Then we have to investigate the identities of such 'deparagrassmannized' (or, as we prefer to say, 'bare') generators, keeping in mind the hope that they will contain some hints about the true structure of the true para-superalgebra and parasupergroup. The complete and rigorous construction of these objects would require a more sharpened technique of handling paragrassmann algebras of many variables than we actually have.

So let us proceed with 'bare' para-generators, which will be denoted by the same letters but in more modest print. Let us concentrate on $G$-generators, for the reason that will be clarified below.

The identities generated by $G$ 's can be described by three following statements:

1. The cyclic bracket of any number of $G$-generators depends only on the product of their arguments:

$$
\frac{1}{M}\left\{G\left(\epsilon_{1}\right), \ldots, G\left(\epsilon_{M}\right)\right\}_{c} \equiv G^{(M)}(\eta), \quad \eta=\epsilon_{1} \cdot \ldots \cdot \epsilon_{M}
$$

2. The similar statement is true for $G^{(M)}$ :

$$
\frac{1}{K}\left\{G^{(M)}\left(\eta_{1}\right), \ldots, G^{(M)}\left(\eta_{K}\right)\right\}_{c}=G^{(K M)}(\zeta), \quad \zeta=\eta_{1} \cdot \ldots \cdot \eta_{K}
$$

3.

$$
G^{(p+1)}(\omega) \equiv T(\omega)
$$

These assertions can be proved by virtue of certain identities in the algebra $\Pi_{p+1}$. The third of them presents the simplest variant of the closure of the algebra generated by $T$ and $G$ by the cyclic bracket, or the cyclator, of $p+1 G$-generators.

The generators $G^{(M)}$ must be treated as the 'bare' variants of $\mathcal{G}^{(M)}$. They have an elegant explicit form

$$
G^{(M)}(\eta)=\eta Q^{M}+\frac{1}{M} \eta^{\prime} \mathcal{J}_{p+1-M}(M=1, \ldots, p+1)
$$

where $\mathcal{J}_{l}$ are certain generators of paragrassmann algebra automorphisms acting as

$$
\mathcal{J}_{l}\left(\frac{\theta^{k}}{\left(\bar{\alpha}_{k}\right) !}\right)=k \frac{\theta^{k+l}}{\left(\bar{\alpha}_{k+l}\right) !} .
$$

Another consequence of (4.6-4.8) is existence of a set of non-equivalent $(p+1)$ linear brackets for composite $p+1$. Really, if $p+1=\nu_{1} \cdot \ldots \cdot \nu_{k}$ for some integer numbers $\nu_{i}$, one can obviously replace $G^{(p+1)}$ in (4.8) by the $\nu_{1}$-linear cyclator of the generators $G^{\left(\nu_{2} \cdots \nu_{k}\right)}$. Then, using 4.7$)$, replace each of $G^{\left(\nu_{2} \cdots \nu_{k}\right)}$ by the $\nu_{2}$-linear 
cyclator of the generators $G^{\left(\nu_{3} \cdots \nu_{k}\right)}$, and so on. As a result one gets a $(p+1)$ linear multi-cyclic bracket of the generators $G$. It is completely determined by the (ordered) sequence $\nu=\left\langle\nu_{1}, \ldots, \nu_{k}\right\rangle$ of the orders of sub-brackets (from outer to inner), and may be labeled by the subscript $\nu$. The subgroup of permutations that leaves the bracket $\{\ldots\}_{\nu}$ invariant will be denoted by $H_{\nu}$. Its order, which coincides with the number of monomials in the bracket, is

$$
N_{\nu}=\nu_{1}\left(\ldots \nu_{k-1}\left(\nu_{k}\right)^{\nu_{k-1}} \ldots\right)^{\nu_{1}}
$$

It is curious to note that this number must be a divisor of $(p+1)$ !.

Brackets corresponding to different sequences $\nu$ are linearly independent. For example, for $p=5$ one can find three 'regular' brackets, corresponding to the sequences $\langle 6\rangle,\langle 2,3\rangle$ and $\langle 3,2\rangle$, which we represent symbolically as $\{123456\},\{\{123\}\{456\}\}$ and $\{\{12\}\{34\}\{56\}\}$ (in this paragraph $\{\ldots\}=\{\ldots\}_{c}$ ). They are evidently independent and containing 6, 18 and 24 terms respectively. Less symmetrical brackets, like $\{\{12\}\{34\}\{5678\}\}$ or like $\{12\} 3+\{23\} 1+\{31\} 2$, can be reduced to sums of several classes of multi-cyclic brackets.

Thus the algebra generated by $T$ and $G$ can be established in general form as

$$
\begin{aligned}
{[T(\omega), T(\eta)] } & =T\left(\omega \eta^{\prime}-\omega^{\prime} \eta\right), \\
{[T(\omega), G(\epsilon)] } & =G\left(\omega \epsilon^{\prime}-\frac{1}{p+1} \omega^{\prime} \epsilon\right), \\
\left\{G\left(\epsilon_{0}\right), G\left(\epsilon_{1}\right), \ldots, G\left(\epsilon_{p}\right)\right\}_{\nu} & =N_{\nu} T\left(\epsilon_{0} \epsilon_{1} \cdots \epsilon_{p}\right) .
\end{aligned}
$$

Introducing the component generators

$$
L_{n}=T\left(z^{-n+1}\right), \quad G_{r}=G\left(z^{-r+1 /(p+1)}\right)
$$

it can be written as

$$
\begin{aligned}
{\left[L_{n}, L_{m}\right] } & =(n-m) L_{n+m}, \\
{\left[L_{n}, G_{r}\right] } & =\left(\frac{n}{p+1}-r\right) G_{n+r}, \\
\left\{G_{r_{0}}, \ldots, G_{r_{p}}\right\}_{\nu} & =N_{\nu} L_{\sum r_{j}} .
\end{aligned}
$$

A particular case of this algebra, when $\nu$ denotes the brackets with all permutations of $G^{\prime}$ 's and $N_{\nu}=\frac{(p+1) !}{p}$, have been presented in the papers [7], [8].

It must be noted that the generators of the algebra (4.10) possess a general representation depending on an arbitrary 'para-conformal' weight $\Delta$ (cf. (1.16)). In the case of Version-(2), the generalization of the formulas (3.47) looks as

$$
\begin{aligned}
T(\omega) & =\omega \partial_{z}+\frac{1}{p+1} \omega^{\prime}\left(\theta \partial_{\theta}+\Delta\right), \\
G(\epsilon) & =\epsilon\left(\partial_{\theta}+\frac{\theta^{p}}{p !} \partial_{z}\right)-\frac{\Delta}{p} \epsilon^{\prime} \frac{\theta^{p}}{p !} .
\end{aligned}
$$

The algebra (4.10), or (4.12), will be called below the paraconformal, or $C_{p}$, and its central extensions will be presented in the next section. 
Before turning to this task we have to make some comments on the $H$-generators. Really, if the algebra $\mathrm{Con}_{p}$ pretends to be a bare form of $\mathcal{C O N}$, it has to deal with all the generators $H_{j}$ and $H_{j}^{(M)}$, as well as with $T, G$ and $G^{(M)}$. The problem is in the following. For generators $G$, the structure of the identities (4.6 - 4.7) and the form of $G^{(M)}$ is practically fixed by the requirement, that the argument of $G^{(M)}$ must be the product of the arguments of the correspondent $G$ 's. For $H$-generators, the similar requirement is almost always fulfilled automatically, and, therefore, it is not clear, which combination should be considered as the right definition of $H^{(M)}$. Then, there exist $p-1$ generators $H_{j}^{(M)}$ in each of $p$ generations $M$, so the number of different identities blows up as $p$ increases. All this makes writing correct relations with $H$-generators a rather subtle problem.

To illustrate the situation, consider the simplest case $p=2$ with one generator $H(\xi)$. The complete paraconformal algebra $\mathrm{Con}_{2}$ may be written as

$$
\begin{aligned}
{[T(\omega), T(\phi)] } & =T\left(\omega \phi^{\prime}-\omega^{\prime} \phi\right), \\
{[T(\omega), G(\epsilon)] } & =G\left(\omega \epsilon^{\prime}-\frac{1}{3} \omega^{\prime} \epsilon\right), \\
{[T(\omega), H(\xi)] } & =H\left(\omega \xi^{\prime}+\frac{1}{3} \omega^{\prime} \xi\right), \\
\{G(\epsilon), G(\zeta), G(\eta)\}_{c} & =3 T(\epsilon \zeta \eta), \\
\{G(\epsilon), G(\zeta), H(\xi)\}_{c} & =G(\epsilon \zeta \xi), \\
\{G(\epsilon), H(\sigma), H(\xi)\}_{c} & =H(\epsilon \sigma \xi) .
\end{aligned}
$$

$G^{(2)}$-generator has the usual form (4.6). $H^{(2)}$-generator can be defined by

$$
G(\eta) H(\xi)+q^{1 / 2} H(\xi) G(\eta)=H^{(2)}(\eta \xi)
$$

so that

$$
G(\zeta) H^{(2)}(\tau)+q^{-1 / 2} H^{(2)}(\tau) G(\zeta)=G(\zeta \tau) .
$$

Here $q$ denotes a primitive cubic root of unity, but this has no connection to the $q$ version. Note that there are no particular reasons for defining $H^{(2)}$ as above, except conciseness of the formulas (4.15) and (4.16).

The cyclic brackets, similar to those in (4.14), also exist for $p>2$ but their number increases with $p$ very fast due to the growing number of $H$-generators. So the problem of a correct description of the $H$-sector in the algebra $C_{0} n_{p}$ looks rather messy. It can hardly be solved without using a Lie-type theory of para-supergroups. For this reason we have excluded the $H$-generators in our treatment of the algebras $\mathrm{Con}_{p}$. The other reason is that the $H$-generators are irrelevant to constructing central extensions, as will be clarified in the next section.

\section{Central Extension of the $\mathrm{Con}_{p}$ Algebra}

A geometric meaning of the algebra $\operatorname{Con}_{p}$ becomes practically obvious after noting that the arguments $\omega, \epsilon$ of the generators (4.13) can be considered not as ordinary functions but as $\lambda$-differentials of suitable weights. The general rule is that generators representing the currents of the spin $s$ (conformal dimension $s$ ) must have the differentials of weight $\lambda=1-s$ as their arguments. So, for $T$ of dimension 2 we 
have $\omega \in \mathcal{F}^{-1}$ and for $G$ of dimension $\frac{p+2}{p+1}$ we have $\epsilon \in \mathcal{F}^{-\frac{1}{p+1}}$. Here and below we denote by $\mathcal{F}^{\lambda}$ the space of the $\lambda$-differentials

$$
\mathcal{F}^{\lambda}=\left\{\omega(z): \omega(z) \mapsto(\tilde{z})^{\lambda} \omega(\tilde{z}), \text { when } z \mapsto \tilde{z}\right\}
$$

The algebra of the generators is then determined by suitable differential operators relating the differentials of different weights. For the generators $T$ and $G$ we may write symbolically

$$
\begin{aligned}
{\left[T\left(\omega_{1}\right), T\left(\omega_{2}\right)\right] } & =T\left(l\left(\omega_{1}, \omega_{2}\right)\right) \\
{[T(\omega), G(\epsilon)] } & =G(m(\omega, \epsilon)) \\
\left\{G\left(\epsilon_{0}\right), \ldots, G\left(\epsilon_{p}\right)\right\}_{\nu} & =T\left(n\left(\epsilon_{0}, \ldots, \epsilon_{p}\right)\right),
\end{aligned}
$$

where the operators $l, m, n$ act as follows:

$$
\begin{aligned}
l: \mathcal{F}^{-1} \Lambda \mathcal{F}^{-1} & \rightarrow \mathcal{F}^{-1} \\
\left(\omega_{1}, \omega_{2}\right) & \mapsto \omega_{1} \omega_{2}^{\prime}-\omega_{1}^{\prime} \omega_{2} \\
l & =d_{2}-d_{1} \\
m: \mathcal{F}^{-1} \Lambda \mathcal{F}^{-\frac{1}{p+1}} & \rightarrow \mathcal{F}^{-\frac{1}{p+1}} \\
(\omega, \epsilon) & \mapsto \omega \epsilon^{\prime}-\frac{1}{p+1} \omega^{\prime} \epsilon \\
m & =d_{2}-\frac{1}{p+1} d_{1} \\
n:\left(\mathcal{F}^{-\frac{1}{p+1}} \times \ldots \times \mathcal{F}^{-\frac{1}{p+1}}\right)_{\nu} & \rightarrow \mathcal{F}^{-1} \\
\left(\epsilon_{0}, \ldots, \epsilon_{p}\right) & \mapsto \epsilon_{0} \cdot \ldots \cdot \epsilon_{p} .
\end{aligned}
$$

Here the symbol $d_{i}$ means differentiating the $i$-th multiplier; the subscript $\nu$ reminds of the symmetry of the bracket.

Let us now turn to central extensions of $\operatorname{Con}_{p}$. Being numbers, central charges can arise from $\lambda$-differentials only as residues of some 1 -forms. Thus, all we have to do to get the central extensions is to find out differential operators acting from the left-hand sides of (5.2) and (5.4) to the sheaf $\mathcal{F}^{1} / d \mathcal{F}^{0}$. The first is the well-known and unique (modulo total derivative) Gelfand-Fuks [19] operator of differential order three: $\phi=d_{1}^{3}-d_{2}^{3}$. The second must be of the order two and have the symmetry group of the bracket, $H_{\nu}$. Thus for the simplest, cyclic bracket we can construct $\left[\frac{p+1}{2}\right]$ operators $\psi_{j}$ :

$$
\psi_{j}=\sum_{i=1}^{p+1} d_{i} d_{i+j}, j=1, \ldots,\left[\frac{p+1}{2}\right] .
$$

Not much more difficult is to construct the operators $\psi_{j}^{(\nu)}$ corresponding to the bracket $\{\ldots\}_{\nu}$ with the symmetry $H_{\nu}$. For instance, the bracket of the type $\{\{123\}\{456\}\}$ admits two operators

$$
\begin{aligned}
\psi_{1} & =d_{1} d_{2}+d_{2} d_{3}+d_{3} d_{1}+d_{4} d_{5}+d_{5} d_{6}+d_{6} d_{4} \\
\psi_{2} & =d_{1} d_{4}+d_{2} d_{4}+d_{3} d_{4}+d_{1} d_{5}+d_{2} d_{5}+d_{3} d_{5} \\
& +d_{1} d_{6}+d_{2} d_{6}+d_{3} d_{6}
\end{aligned}
$$


And so on. The larger is the symmetry group of the bracket, $H_{\nu}$, the smaller is the number of admissible central charges $E_{\nu}$. For the regular brackets

$$
E_{\nu}=\sum_{k}\left[\frac{\nu_{k}}{2}\right]
$$

The resulting extended algebra is

$$
\begin{aligned}
{\left[T\left(\omega_{1}\right), T\left(\omega_{2}\right)\right] } & =T\left(\omega_{1} \omega_{2}^{\prime}-\omega_{1}^{\prime} \omega_{2}\right)+C \phi\left(\omega_{1}, \omega_{2}\right) \\
{[T(\omega), G(\epsilon)] } & =G\left(\omega \epsilon^{\prime}-\frac{1}{p+1} \omega^{\prime} \epsilon\right) \\
\left\{G\left(\epsilon_{0}\right), \ldots, G\left(\epsilon_{p}\right)\right\}_{\nu} & =N_{\nu} T\left(\epsilon_{0} \cdot \ldots \cdot \epsilon_{p}\right)+\sum_{j=1}^{E_{\nu}} c_{j} \psi_{j}^{(\nu)}\left(\epsilon_{0}, \ldots, \epsilon_{p}\right),
\end{aligned}
$$

where $N_{\nu}$ is the number of the terms in the bracket. The central charge $C$ can be expressed in terms of $c_{j}$ by commuting the third line of Eq. (5.7) with some $T(\eta)$

and then comparing both sides of the resulting identity. Let us apply this procedure to the cyclic bracket. Writing the generators in components we get the algebra announced in the Introduction as $\operatorname{Vir}_{p}$ :

$$
\begin{array}{rll}
{\left[L_{n}, L_{m}\right]} & =(n-m) L_{n+m}+\frac{2}{p+1}\left(\sum_{j} c_{j}\right)\left(n^{3}-n\right) \delta_{n+m, 0} & \\
{\left[L_{n}, G_{r}\right]} & =\left(\frac{n}{p+1}-r\right) G_{n+r} & j=1, \ldots,\left[\frac{p+1}{2}\right] \\
\left\{G_{r_{0}}, \ldots, G_{r_{p}}\right\}_{c} & =(p+1) L_{\Sigma r}-\sum_{j} c_{j}\left(\sum_{i} r_{i} r_{i+j}+\frac{1}{p+1}\right) \delta_{\Sigma r, 0} &
\end{array}
$$

Note that the symmetry of the extension operators may be taken wider than that of the bracket. That would be equivalent to constraining some of the charges $c_{j}$. For example, there exists a unique totally symmetric operator $\Psi=\sum_{i<j} d_{i} d_{j}$ that can be used with all kinds of brackets. Unfortunately, this simple extension seems to be unsuitable for constructing a non-trivial analog of the Verma module.

\section{Discussion and Conclusion}

Let us summarize the results and problems beginning with the results.

1. We have introduced the version-covariant fractional derivative, the inverse operator (integral), and the Taylor expansion. This demonstrates that the fractional derivative has many properties in common with the superderivative and so may be indeed regarded as its generalization.

2. Transformations of the para-superplane preserving the form of the fractional derivative $\mathcal{D}$ obey the transitivity condition and form a group $\mathbf{C O N}_{p}$ that is called the paraconformal group. Simplest examples of the global paraconformal transformations are given in Appendix.

3. The corresponding infinitesimal object, a 'true' paraconformal algebra $\mathcal{C O N}_{p}$, is related to the space of $p$-jets rather than to the tangent space. $\mathcal{C O N}_{p}$ is a $p$ filtered algebra with generators in $p$ generations. Generators of $M$-th generation do not occur in the order of smallness less than $M$.

The generators of the first generation are: the usual conformal generator $T$ with the conformal weight 2 , the paraconformal generator $G$ with the weight $\frac{p+2}{p+1}$, and 
the paragrassmann generators $\mathcal{H}_{j}(j=1 \ldots p-1)$ with the weights $\frac{p+1-j}{p+1}$. The generators of the $M$-th generation are: $\mathcal{G}^{(M)}$ with the weight $1+\frac{M}{p+1}$ and $\mathcal{H}_{j}^{(M)}$ with the weight $\frac{p+M-j}{p+1}$. The $\mathcal{H}$-type generators do not affect $z$-coordinate of the para-superplane $(z, \theta)$ but they are required by self-consistency of the algebra.

4. Algebra $\mathcal{C O N}_{p}$ can be considered as a paragrassmann shell of a 'bare' ('skeleton') algebra $C n_{p}$ also called paraconformal. Its generators $T, G^{(M)}, H_{j}^{(M)}$ have as their arguments ordinary functions (in fact, $\lambda$-differentials). The connection between $\mathcal{C O N}_{p}$ and $\mathrm{Con}_{p}$ is trivial in the supercase $(p=1)$ but it is not so clear for $p>1$. We have systematically derived identities in the algebra $C_{0} n_{p}$ that must encode some information about the structure of the algebra $\mathcal{C O} \mathcal{N}_{p}$ but understanding of exact relations between these two algebras is still lacking.

5. The algebra $C_{0} n_{p}$ can be closed in terms of $T$ - and $G$-generators only (unlike $\mathcal{C O} \mathcal{N}_{p}$ ). There exist many multilinear identities with $G$-generators based on the cyclic brackets of arbitrary order. For composite $p+1$, they give rise to a set of nonequivalent $(p+1)$-linear brackets of $G$ closing to $T$. This, by the way, makes evidence that the algebra $C_{0} n_{p}$ contains as a subalgebra $\operatorname{Con}_{r}$ when $(r+1)$ is a divisor of $(p+1)$ (in view of drastic simplifications of the paragrassmann calculus and of the paraconformal transformations occurring for prime integer $p+1$, a detailed further treatment of this case is an obvious priority).

6. To each of these brackets there corresponds a set of basic central extension operators having the same symmetry as the bracket. The wider is the symmetry, the smaller is the number of extensions. Constraining the coefficients (the central charges), one can enlarge the symmetry of the central term as compared to the bracket.

Here emerges a branching point for future development.

One way is to consider different brackets (and the identities of smaller order as well) just as the identities in the same algebra $\mathrm{Con}_{p}$, and then to deal with the unique central extension that suits all of them. This corresponds to the extension generated by the totally symmetric operator $\Psi$.

The other way is to forget all preliminaries about the infinitesimal generators and paragrassmann algebras and to consider the algebras of $T$ and $G$ with different brackets as independent infinite-dimensional algebras, each having its own central extension. This approach might appear fruitful for simple brackets, like the cyclic ones. A right way is probably somewhere in between and hence the problem of a lucky choice of the symmetry breaking arises.

We think that 'the right way' is that leads to a nontrivial Verma module. In fact, a natural program to develop the theory is to define a suitable analog of a Verma module over the algebra $V i r_{p}$ and to search for degenerate modules, Kac determinant and rational models. Unfortunately even first steps appear to be nontrivial. Let us illustrate the problem by a simple example.

Consider the algebra $\mathrm{Vir}_{2}$ with the third line being taken with a totally symmetric bracket:

$$
\left\{G_{r}, G_{s}, G_{t}\right\}_{s y m}=6 L_{r+s+t}+C\left(r^{2}+s^{2}+t^{2}\right) \delta_{r+s+t, 0}
$$


Assume that the constraint $F_{r+s}=\left\{G_{r}, G_{s}\right\}$ of the algebra Con $_{2}$ is preserved. Then for any acceptable positive $k$ we can write two strings

$$
\begin{gathered}
F_{\frac{2 k}{3}} G_{-\frac{2 k}{3}}+2 F_{-\frac{k}{3}} G_{\frac{k}{3}}=6 L_{0}+\frac{2}{3} C\left(k^{2}-1\right), \\
2 F_{\frac{2 k}{3}} G_{-\frac{2 k}{3}}+F_{-\frac{4 k}{3}} G_{\frac{4 k}{3}}=6 L_{0}+\frac{2}{3} C\left(4 k^{2}-1\right) .
\end{gathered}
$$

Now defining a vacuum so that

$$
G_{>0} \approx 0, \quad L_{0} \approx \Delta
$$

(we write $X \approx Y$ for $X|v a c\rangle=Y|v a c\rangle$ ) we immediately get a contradiction,

$$
F_{\frac{2 k}{3}} G_{-\frac{2 k}{3}} \approx 6 \Delta+\frac{2}{3} C\left(k^{2}-1\right) \approx \frac{1}{2}\left(6 \Delta+\frac{2}{3} C\left(4 k^{2}-1\right)\right),
$$

unless both $\Delta$ and $C$ are zero. This is an evidence of a rather general phenomenon. Namely, preserving constraints while keeping to a naive definition of the vacuum is, as a rule, inconsistent with a nontrivial central charge (and often with a nontrivial highest weight, as in the example). To bypass this disaster one might either try to select an appropriate set of the constraints to be preserved or to redefine the vacuum in a more skillful way. Our attempts in this direction have not produced anything valuable so far.

One might also suspect the $H$-generators might play a role in defining the vacuum and the module. However, the above example gives little support to this suspicion.

Some light on the topic might be thrown by investigating concrete physical systems possessing paraconformal symmetry. But the algebraic results of the present paper are hinting that quantizing such systems will be rather ambiguous.

Thus, the current problems may be summarized in the following list:

1. The main theoretical problem is to find a rigorous connection between the three constructed paraconformal objects: $\mathbf{C O N}_{p}, \mathcal{C O N}_{p}$ and $C o n_{p}$. This problem requires further developing the calculus for many paragrassmann variables.

2. It is not clear how to correctly include the $H$-generators into the algebras $\mathrm{Con}_{p}$ and $\operatorname{Vir}_{p}$.

3. The main practical question is to find a non-trivial Verma module over $V_{i r}$.

Ending, we would like to note that paraconformal algebras are not of a pure aesthetic interest. For example, on a Riemann surface of genus $g$ the natural scale of conformal dimension is $\frac{1}{2(g-1)}$, as a consequence of the Gauss-Bonnet theorem, and thus the natural fractional derivative is of the same order, and the natural conformal algebra would be $V i r_{2 g-3}$ rather than $V i r_{1}=R N S$. One may also speculate that using paraconformal algebras might drastically change the critical dimensions in the string theory.

\section{Appendix}

Here we present paraconformal transformations generalizing translations, inversions and some other superconformal transformations. To simplify formulas we consider the case $p=2$. 
Let us try to write para-translations assuming $\tilde{\theta}=\theta+\epsilon_{0}$, where $\epsilon_{0}$ is a paragrassmann number (independent of $z$ and satisfying $\epsilon_{0}^{3}=0$ ). The functions $Z_{i}$ can be found from Eqs.(3.21). By applying the relation $[\tilde{z}, \tilde{\theta}]=0$ and Eq.(3.19) we can find the commutation relation $(\overline{3.33})$ and

$$
\alpha_{2}\left(\alpha_{2}-1\right) \theta \epsilon_{0}^{2}=\partial\left(\epsilon_{0}^{2} \theta^{2}\right)
$$

that immediately give

$$
\alpha_{2}^{2}-\alpha_{2}+1=0
$$

This shows that either $\alpha_{2}=-q$ or $\alpha_{2}=-q^{2}$, where $q$ is the prime cubic root of unity. With $\alpha_{2}=-q^{2}=1+q$ and choosing $k=1$ in Eq.(3.2), the translations can be written in the form

$$
\tilde{z}=z+z_{0}-q\left(\theta \epsilon_{0}^{2}+\theta^{2} \epsilon_{0}\right), \tilde{\theta}=\theta+\epsilon_{0} .
$$

If we further assume that $\epsilon_{0} \theta=q \theta \epsilon_{0}$ thus automatically satisfying Eq.(3.33), we can find the operator generating translations $\left(\bar{\alpha}_{2}=-q=1+\bar{q}\right)$

$$
\begin{gathered}
\mathcal{G}\left(z_{0}, \epsilon_{0}\right)=e^{z_{0} \partial_{z}}\left(1+\epsilon_{0} Q+\bar{\alpha}_{2}^{-1} \epsilon_{0}^{2} Q^{2}\right), \\
\mathcal{G}\left(z_{0}, \epsilon_{0}\right) F(\theta, z)=F(\tilde{\theta}, \tilde{z}),
\end{gathered}
$$

where $Q$ is defined by Eq.(3.39). One can check that all conditions defining paraconformal transformations are satisfied for the translations.

We may now apply these results to the integrals in Eqs.(3.5), (3.6). Assuming $\epsilon_{0} \theta_{i}=q \theta_{i} \epsilon_{0}$ where $i=1,2$ it is easy to show that $I_{2}$ and $I_{3}$ are invariant under translations (invariance of $I_{1}$ is obvious). However, this does not give a naive generalization of the properties of the Taylor expansion in the Grassmann case. One can show that the integrals $I_{n}$ can be simply expressed in terms of $I_{1}, I_{2}, \bar{I}_{3}=I_{3}-\left(z_{1}-z_{2}\right) / k$, of powers of $\left(z_{1}-z_{2}\right) / k$, and of one additional non-invariant expression $\theta_{1}^{2} \theta_{2}^{2}$. The transformation properties of the coefficients in the Taylor expansion (3.5) are thus not as obvious as in the Grassmann case. Possibly, our definition of the translations is not quite suitable for this expansions. In this connection, we note that other definitions of translations are possible but they require specifying many-variable paragrassmann calculus which we consistently avoid in this paper.

Finally, let us write more general paraconformal transformations. With the above assumptions of $q$-commutativity between $\theta$ and the paragrassmann parameters, it is not difficult to show that the following transformation satisfies all necessary conditions:

$$
\begin{gathered}
\tilde{\theta}=\epsilon_{1}+\left(z / z_{2}\right)^{\lambda} \theta ; \\
\tilde{z}=\left[z_{1}+z_{2}(3 \lambda+1)^{-1}\left(z / z_{2}\right)^{3 \lambda+1}\right]-q\left(z / z_{2}\right)^{\lambda} \theta \epsilon_{1}^{2}-q\left(z / z_{2}\right)^{2 \lambda} \theta^{2} \epsilon_{1} .
\end{gathered}
$$

Here $\lambda, z_{1}, z_{2}$ are complex parameters, $\epsilon_{1}$ - a paragrassmann number that $q$ commutes with $\theta$, like $\epsilon_{0}$. For $\lambda=0$ we return to the above translations. Applying translations to the general transformations depending on arbitrary $\lambda$, we obtain rather general paraconformal transformations depending on four complex numbers $\lambda, z_{0}, z_{1}, z_{2}$ and on two paragrassmann parameters $\epsilon_{0}$ and $\epsilon_{1}$. With $\lambda=-2 / 3$ 
one can then derive a natural para-extension of the $S L(2, C)$ transformation. The above transformations may be further generalized if we replace the power function in the square brackets by an arbitrary function of $z$ and express other powers of $z$ in terms of its derivative. We think that these explicit formulas show that the parasuperplane is a rich but not hopelessly complicated thing worth of further study.

\section{Acknowledgments}

This paper has been completed while one of the authors (A.T.F.) visited Yukawa Institute of Theoretical Physics. A.T.Filippov wishes to express his deep gratitude to Y.Nagaoka and T.Inami for a very kind hospitality and support. Useful comments by A.LeClair, S.Novikov, V.Spiridonov and S.Pakuliak are also acknowledged.

\section{References}

[1] A.B.Zamolodchikov, Rev. Math. Phys., 1 (1990)197;

L.Alvarez-Gaume, C.Gomez and G.Sierra, in: Physics and Mathematics of Strings, Eds. L.Brink, D.Friedan and A.Polyakov, World Sci., Singapore, 1990.

[2] V.G.Knizhnik, Theor. Math. Phys., 66 (1986)68;

M.Bershadsky, Phys. Lett., 174B (1986)285;

A.Schwimmer and N.Seiberg, Phys. Lett. 184B (1987)191;

E.Ivanov, S.Krivonos and V.Leviant, Nucl. Phys. B304 (1988)601.

[3] V.A.Fateev and A.B.Zamolodchikov, Sov. Phys. JETP 62 (1985)215;

Theor. Math. Phys., 71 (1987)451.

[4] V.A.Rubakov and V.P.Spiridonov, Mod. Phys. Lett. A3 (1988)1337;

V.P.Spiridonov, J. Phys. A24 (1991) L529.

[5] S.Durand, R.Floreanini, M.Mayrand and L.Vinet, Phys. Lett. 233B (1989)158.

[6] Y.Ohnuki and S.Kamefuchi, Quantum Field Theory and Parastatistics, Univ. of Tokyo Press, 1982;

A.B.Govorkov, Sov. J. Part. Nucl. 14 (1983)520.

[7] S.Durand, Fractional Superspace Formulation of Generalized Super-Virasoro Algebras, preprint McGill Univ. 92-30, 1992; hep-th 9205086.

[8] A.T.Filippov, A.P.Isaev and A.B.Kurdikov, Mod. Phys. Lett. A7 (1992)2129.

[9] C.Ahn, D.Bernard and A.LeClair, Nucl. Phys. B346 (1990)409.

[10] T.Nakanishi, Progr. Theor. Phys. 82 (1989)207. 
[11] D.Friedan, Notes on String Theory and Two Dimensional Conformal Field Theory, in: Proceedings of the Workshop on Unified String Theories, Santa Barbara, 1985;

M.A.Baranov and A.S.Schwarz, JETP Lett. 42 (1986)419.

[12] M.A.Baranov, I.V.Frolov and A.S.Schwarz, Theor. Math. Phys. 79 (1987) 64; Ph.Nelson, Lectures on Supermanifolds and Strings, in: Particles, Strings and Supernovae, Eds. A.Jevicki and C.I.Tan W.Sci., Singapore, 1988;

C.Itzykson and J.-M.Drouffe, Statistical Field Theory, Cambridge Univ. Press, Cambridge, 1989.

[13] A.T.Filippov, A.P.Isaev and A.B.Kurdikov, On Paragrassmann Differential Calculus, preprint JINR E5-92-392, Dubna, 1992;

Paragrassmann Differential Calculus, hep-th 9210075, preprint YITP/K-995, 1992, to be published in Theor. Math. Phys..

[14] S.Durand, R.Floreanini, M.Mayrand, V.Spiridonov and L.Vinet, Parasupersymmetries and Fractional Supersymmetries, in: Proceedings of the XVIII-th International Colloquium on Group Theoretical Methods in Physics, Moscow, 1990.

[15] N.Jacobson, Lie Algebras, Interscience Publ., N.-Y.-London, 1962.

[16] A.MacFarlane, J. Phys. A22 (1989)4581;

L. Biedenharn, ibid. A22 (1989)L873.

[17] P.Brocker and L.Lander, Differential Germs and Catastrophes, Cambridge Univ. Press, Cambridge, 1975.

[18] V.V.Vishnevskii, A.P.Shirokov and V.V.Shurygin, Spaces over Algebras, Kazan University Publ., Kazan, 1985 (in Russian).

[19] I.M.Gelfand and D.B.Fuks, Func. Anal. Appl. 3 (1969) 194; 4 (1970) 110. 